\title{
A THEORY OF ABSOLUTELY CONTINUOUS TRANSFORMATIONS IN THE PLANE
}

\author{
BY \\ T. RADÓ AND P. REICHELDERFER
}

\section{Chapter I. General outline of the theory}

1.1. The concept of an absolutely continuous function of a single real variable plays a fundamental rôle in a variety of one-dimensional problems in geometry and analysis. Many efforts have been made to develop an equally useful concept of two-dimensional absolute continuity. The purpose of the present paper is to make a contribution to this field through a comprehensive study of a new type of absolute continuity for continuous transformations in the plane $\left.{ }^{1}\right)$. In view of the number and variety of concepts and results, it seems advisable to present first a detailed outline of the theory, in order that the reader may more easily obtain a general idea of it. Thus this chapter contains statements of the fundamental definitions and of the more important theorems, as well as some remarks concerning the relationship of this theory to previous literature. In order to avoid duplications, the rest of the paper consists essentially of concise proofs of the results stated in this chapter.

1.2. We commence with a few remarks concerning the very extensive relevant literature $\left({ }^{2}\right.$ ) (see Banach [1, 2], Bray [1], Caccioppoli [2], McShane [1], Morrey [1], Rademacher [1], Radó [5], Schauder [1], Young [1, 2, 3]). For definiteness, let us consider a continuous transformation $T$ of the form

$$
T: \quad x=x(u, v), \quad y=y(u, v),
$$

where $x(u, v), y(u, v)$ are defined and continuous in the unit square

$$
S: \quad 0 \leqq u \leqq 1, \quad 0 \leqq v \leqq 1 .
$$

Two essentially different types of two-dimensional absolute continuity have thus far been considered in the literature in the study of such a transformation. The first type, due to Banach (see Banach [2]), is a direct generalization of the concept of an absolutely continuous function $x=x(u)$ of a single real variable (where the relation $x=x(u)$ is interpreted as defining a continuous one-dimensional transformation). If $E$ is any set in the square $S$ and if $T(E)$

Presented to the Society, April 15, 1939, under the title Some properties of continuous transformations in the plane; received by the editors June 6, 1940.

(1) The class of transformations which are absolutely continuous, in our sense, in a domain $\mathcal{D}$ will be denoted by $K_{1}(\mathcal{D})$, and will be described in 1.32 . Important subclasses of $K_{1}(\mathcal{D})$ will be defined in $\$ 1.34$ and $\$ 1.37$.

(2) Numbers in square brackets are used to refer to the references listed in the bibliography at the end of this paper. 
denotes the image of $E$ in the $x y$ plane, then define $\left({ }^{3}\right)$

$$
\Psi(E)=|T(E)| \text {. }
$$

If there exists a finite constant $M$ such that

$$
\sum \Psi\left(s_{i}\right)<M
$$

for every finite sequence of nonoverlapping, closed, oriented $\left({ }^{4}\right)$ squares $s_{i}$ in $S$, then $T$ is of bounded variation in the sense of Banach. If, for every $\epsilon>0$ there exists an $\eta=\eta(\epsilon)>0$ such that

$$
\sum \Psi\left(s_{i}\right)<\epsilon
$$

for every finite sequence of nonoverlapping, closed, oriented squares $s_{i}$ in $S$ satisfying

$$
\sum\left|s_{i}\right|<\eta
$$

then $T$ is absolutely continuous in the sense of Banach. For transformations $T$ absolutely continuous in this sense, Banach (see Banach [2]) and Schauder (see Schauder [1]) developed a comprehensive and very beautiful theory which has since been completed in many respects by Radó (see Radó [5]). While this theory is wholly satisfactory from the aesthetic point of view, it is a fact that the underlying concept of absolute continuity is too restrictive to permit extensive applications. Indeed, many important classes of continuous transformations, which have been studied by various mathematicians (cf. $\$ 1.3$ ) in recent years, do not possess the type of absolute continuity required by Banach.

1.3. In a majority of recent investigations in this field, the assumptions concerning $T$ read as follows.

(i) The functions $x(u, v), y(u, v)$ are both absolutely continuous in the sense of Tonelli $\left({ }^{5}\right)$.

(ii) Further assumptions, concerning $x(u, v), y(u, v)$ jointly, which we do not have to state. We observe only that these assumptions vary considerably, both in character and in generality.

In contradistinction to the type of absolute continuity described in 1.2 , the above condition (i) requires, so to speak, iterated linear absolute continuity

( $\left.{ }^{3}\right)$ If $e$ be any set, then $|e|$ will denote the exterior measure of $e$.

(4) A rectangle $R$, or a square $S$, is termed oriented when its sides are parallel to the respective coordinate axes. Only such squares and rectangles are considered in this paper.

(5) The function $x(u, v)$, for example, is absolutely continuous in the sense of Tonelli if (i) $x(u, v)$ is of bounded variation in the sense of Tonelli, and (ii) for almost every $v=\eta, 0 \leqq \eta \leqq 1$, the function $x(u, \eta)$ is absolutely continuous in $u$, and, for almost every $x=\xi, 0 \leqq \xi \leqq 1$, the function $x(\xi, v)$ is absolutely continuous in $v$. Denote by $V(\eta)$ the total variation of $x(u, \eta)$ as a function of $u$ on $0 \leqq u \leqq 1$; denote by $V(\xi)$ the total variation of $x(\xi, v)$ as a function of $v$ on $0 \leqq v \leqq 1$. Then $x(u, v)$ is of bounded variation in the sense of Tonelli if $V(\eta)$ and $V(\xi)$ are summable functions of $\eta$ and $\xi$ respectively on the interval $(0,1)$. 
of $x(u, v)$ and $y(u, v)$ separately. While results of great importance and generality have been derived from assumptions of this character, it is also a fact that for several of the most advanced results, it has been shown that the assumption (i) above either can be replaced by bounded variation in the sense of Tonelli $\left({ }^{6}\right)$, or can be discarded entirely (see Radó $[3,4]$ ).

1.4. The preceding remarks suggest that the concepts of absolute continuity described in $\$ 1.2$ and $\$ 1.3$ may not represent the ultimate in usefulness. The purpose of the present paper is to develop, for continuous transformations in the plane, a theory of absolute continuity which is, in the first place, as truly two-dimensional as the theory initiated by Banach, and which is, in the second place, sufficiently comprehensive to permit us to account for and to improve upon the major results obtained for classes of transformations of the character described in $\$ 1.3$.

1.5. The basic idea in our theory is to replace, in the definition of absolute continuity due to Banach (cf. $\$ 1.2$ ), the function of squares $\Psi(s)=|T(s)|$ by a (generally) smaller function, making thereby the requirement of absolute continuity less restrictive, and hence the class of absolutely continuous transformations more comprehensive. Intuitively speaking, we shall replace the measure of the image of the square $s$ by the measure of the essential part of that image. This idea was derived by Rado from a study of the profound and involved work of Geöcze on the area of continuous surfaces (see Radó [1] for references). After the publication in 1928 of the first applications of this idea (see Radó [1]), essentially the same idea was used in 1930 by Caccioppoli (see Caccioppoli [2]) to develop a theory of absolutely continuous transformations in the plane; he applied his theory, in a series of subsequent papers, to a variety of fundamental problems involving double integrals. Since the work of Caccioppoli is based essentially upon the same original ideas and is pointed toward the same general objectives as our work, the following general remarks seem important.

1.6. The theory with which we are concerned is, fundamentally, a metric theory of topologically defined set functions. Such set functions are, as a rule, directly defined only for certain simple sets. A basic feature of the work of Caccioppoli is the additive extension of the range of definition of these set functions to the class of all Borel sets (at least). He infers the possibility of such extensions from one of his theorems which may be described as follows (see Caccioppoli [1]).

Let there be given a function $\Phi$ defined for all open sets comprised, say, in a fixed rectangle $R$ and satisfying the following conditions : (i) $\Phi(O) \geqq 0$ for every $O \subset R$; (ii) if $O_{1}, O_{2}, \cdots$ are nonoverlapping open sets in $R$, then $\Phi\left(\sum O_{n}\right)=\sum \Phi\left(O_{n}\right)$; (iii) if $O^{\prime} \subset O^{\prime \prime} \subset R$, then $\Phi\left(O^{\prime}\right) \leqq \Phi\left(O^{\prime \prime}\right)$; (iv) if $O_{\epsilon}$ denotes the (open) set of those points in $O$ (where $O$ is any open set in $R$ ) whose dis-

${ }^{(6)}$ See $\left({ }^{5}\right)$. 
tance from the boundary of $O$ exceeds $\epsilon$, then $\lim \Phi\left(O_{\epsilon}\right)=\Phi(O)$ as $\epsilon$ tends to zero, for every choice of $O \subset R$. According to Caccioppoli, it should then always be possible to extend the definition of $\Phi$ to all Borel sets in $R$ in such a way that the extended function is completely additive on the class of all Borel sets in $R$.

1.7. Unfortunately, the preceding theorem is false, as the reader will easily verify by considering the following example: let $S$ denote a fixed closed square in the interior of $R$; define $\Phi(O)=1$ if $O \supset S$, and $\Phi(O)=0$ otherwise. Not only is this general extension theorem of Caccioppoli false, but it is also a fact that the particular set functions which he proposes to extend additively cannot generally be so extended. Since many of his basic definitions are stated in terms of (generally non-existing) additive extensions, we were unable, notwithstanding considerable effort, to understand the details of his theory. However, it would seem that the fundamental error pointed out above prevented Caccioppoli from recognizing essential difficulties in the situationdifficulties which delayed the completion of our own work for several years $\left({ }^{7}\right)$.

1.8. We now proceed to give a summary of the contents of this paper. Let $S$ denote a bounded point set in the $u v$-plane. A pair of (real-valued) functions $x(u, v), y(u, v)$, bounded and continuous on $S$, determines a continuous transformation

$$
T: \quad x=x(u, v), \quad y=y(u, v), \quad(u, v) \in S .
$$

The symbol $\in$ will be used to mean "is an element of the set." For conciseness, we shall also use the complex notation $z=x+i y, w=u+i v$, and write

$$
T: \quad z=t(w), \quad w \in S,
$$

where $t(w)$ is then bounded and continuous on $S$. We term $T$ a bounded continuous transformation defined on $S$. If $E$ is any set in the w-plane, then $T(E)$ will denote the image of the set $E \cdot S$ under $T$ in the $z$-plane. If $\bar{E}$ is any set in the z-plane, then $T^{-1}(\bar{E})$ will denote the set of all those points $w \in S$ whose image is in $\bar{E}$; this set is termed the inverse of the set $\bar{E}$ under $T$.

1.9. If we are given a second bounded continuous transformation

$$
T_{*}: z=t_{*}(w), \quad w \in S,
$$

and if $E$ is any set in the w-plane, then we define

$$
\rho\left(T_{*}, T ; E\right)=\left\{\begin{array}{l}
\text { l.u.b. }\left|t_{*}(w)-t(w)\right| \text { for } w \in E \cdot S, \text { if } E \cdot S \neq 0, \\
0 \text { if } E \cdot S=0 .
\end{array}\right.
$$

This quantity $\rho\left(T_{*}, T ; E\right)$ will be called the distance of $T_{*}$ and $T$ on the set $E$.

( $\left.{ }^{7}\right)$ A detailed analysis of fundamental portions of Caccioppoli's work is contained in the (as yet unpublished) Ohio State University Dissertation, Some properties of continuous transformations in the plane, 1939, by Paul V. Reichelderfer. 
Clearly this distance is symmetric, satisfies the triangle inequality, and vanishes if and only if $t_{*}(w)=t(w)$ on $E \cdot S$ (provided $E \cdot S$ is not empty).

1.10. We shall use the symbol $N(z, T, E)$ to denote the number (possibly equal to $+\infty)$ of distinct points $w$ in the set $E \cdot T^{-1}(z)$. The set of those points $z$ for which $N(z, T, E)=+\infty$ will be denoted by $\widetilde{\mathfrak{S}}(\infty, T, E)$.

1.11. The symbol $\Re$ will be used to denote generically a (bounded, finitely connected) Jordan region $\left({ }^{8}\right)$. Given a bounded continuous transformation $T$ defined on a Jordan region $\Re$ in the w-plane (cf. $\$ 1.8$ ), and a non-negative integer $k$, we define the set $\bar{\AA}(k, T, \Re)$-termed the kernel $\left({ }^{9}\right)$ of order $k$ of the image of $\Re$ under $T$ (see Radó [5, p. 202]) —as the set of those points $z$ for each of which there exists a number $\epsilon=\epsilon(k, z, T, \Re)>0$ such that, for every continuous transformation $T_{*}$ defined on $\Re$ and satisfying the inequality $\rho\left(T_{*}, T ; \Re\right)<\epsilon($ cf. $\$ 1.9)$, it is true that $N\left(z, T_{*}, \Re\right) \geqq k$ (cf. $\left.\$ 1.10\right)$. Clearly a kernel of order $k>0$ is a subset of every kernel of lower order. Next, we set $\left({ }^{10}\right)$

$$
\bar{\Omega}(\infty, T, \Re)=\prod_{k=0}^{\infty} \bar{\Re}(k, T, \Re) .
$$

This set will be called the kernel of order $+\infty$. Some, or all of the kernels of order $k \geqq 1$ may be empty.

1.12. In the $z$-plane, we now define a function $\kappa(z, T, \Re)$-called the essential multiplicity of the point $z$ in the image of $\Re$ under $T$-as follows (see Radó [5, p. 205]):

$$
\kappa(z, T, \Re)=\left\{\begin{array}{l}
k \text { for } z \in \overline{\widehat{\Re}}(k, T, \Re)-\bar{\AA}(k+1, T, \Re), \\
+\infty \text { for } z \in \bar{\Omega}(\infty, T, \Re) .
\end{array}\right.
$$

Obviously $\kappa(z, T, \Re) \leqq N(z, T, \Re)$. While $N(z, T, \Re)$ apparently possesses no useful continuity properties, we shall see (cf. $\$ 2.5)$ that $\kappa(z, T, \Re)$ is a lower semi-continuous function of its arguments $z$ and $T$.

1.13. Next, we consider a bounded domain $\left({ }^{11}\right) \mathcal{D}$ in the w-plane, and on it a bounded continuous transformation (cf. \$1.8)

$$
T: \quad z=t(w), \quad w \in \mathcal{D} .
$$

If a sequence of Jordan regions $\Re_{n}$ is such that (i) $\Re_{n} \subset \mathcal{D}$, and (ii) for every closed set $F \subset \mathcal{D}$ there exists an $n_{0}=n_{0}(F)$ such that $F \subset \Re_{n}$ for all $n>n_{0}$, then we shall say that the Jordan regions $\Re_{n}$ fill up $\mathcal{D}$ from the interior. For such

$\left.{ }^{8}\right)$ Since the terms region and domain do not seem to possess standardized meanings, we agree on the following terminology. A domain is a connected open set, while a region consists of a connected open set plus its boundary.

( $\left.{ }^{9}\right)$ For $k=1$ this concept was proposed and studied in Rad6 [1].

(10) Since our definition of the kernel of a finite order would remain meaningful for $k=+\infty$, it is important to note that we use an entirely different definition for the kernel of order $+\infty$.

(ii) See $\left.{ }^{8}\right)$. 
a sequence of Jordan regions we shall see (cf. \$2.6) that the sequence $\kappa\left(z, T, \Re_{n}\right)$ converges for every choice of $z$, the limit being possibly equal to $+\infty$. The limit is then clearly independent of the choice of the Jordan regions which fill up $\mathcal{D}$ from the interior. We define

$$
\kappa(z, T, \mathcal{D})=\lim _{n \rightarrow \infty} \kappa\left(z, T, \Re_{n}\right) .
$$

This function $\kappa(z, T, \mathcal{D})$-termed the essential multiplicity of the point $z$ in the image of $\mathcal{D}$ under $T$-is fundamental in our work. To state one of our principal results, we need the following concepts.

1.14. Let $\Re$ be a Jordan region comprised in $\mathcal{D}$. Let $C$ be one of the boundary curves of $\Re$ and let $z$ be a point in the $z$-plane. As a point $w$ describes $C$ once in the positive sense with respect to $\Re$, the point $t(w)$ describes a directed closed continuous curve in the $z$-plane, which we shall denote by $\bar{C}$. We define $\mu(z, T, C)$ as the topological index (see Kerékjártó [1] or Radó [5]) of the point $z$ with respect to $\bar{C}$ if $z$ is not on $\bar{C}$; if $z$ is on $\bar{C}$, we put $\mu(z, T, C)=0$. Finally, if $z$ is not on the image, under $T$, of the boundary of $\Re$, we define $\mu(z, T, \Re)=\sum \mu(z, T, C)$, the summation being extended over all the boundary curves of $\Re$; we set $\mu(z, T, \Re)=0$ if $z$ is on the image, under $T$, of the boundary of $\Re$.

1.15. If $\mu(z, T, \Re) \neq 0$, then we shall say that $\Re$ is an indicator region for the point $z$ under $T$, or briefly, an indicator region $(z, T)$. An indicator system $(z, T)$ of order $k$ is defined as a system of $k$ nonoverlapping indicator regions $(z, T)$.

1.16. Given a point $z$ in the $z$-plane, let us consider a component of its inverse $T^{-1}(z)$ (cf. $\$ 1.8$ ). Such a component may or may not be a continuum (although it is always a closed set relative to $\mathcal{D}$ ); but if it is, we shall call it a maximal model continuum of $z$ under $T$ in $\mathcal{D}$ and we shall denote it generically by $\sigma(z, T)$. A $\sigma(z, T)$ will be called essential if every open set which contains it also contains a Jordan region $\Re$ which is an indicator region $(z, T)$ and which contains $\sigma(z, T)$ (necessarily in its interior).

1.17. We have then the fundamental theorem( ${ }^{12}$ ) (cf. \$2.6):

$\kappa(z, T, \mathcal{D})$ is equal to the number of distinct essential maximal model continua of $z$ under $T$ in $D$.

1.18. Given a bounded continuous transformation $T$ defined on a bounded domain $\mathcal{D}$ (cf. $\S 1.13$ ), we define a subset $\mathcal{E}=\mathcal{E}(T, \mathcal{D})$ of $\mathcal{D}$ as follows. A point $w_{0} \in \mathcal{D}$ belongs to $\mathcal{E}$ if $w_{0}$ itself constitutes an essential maximal model continuum of its image $t\left(w_{0}\right)$. We define further a subset $\mathcal{N}=\mathcal{N}(T, \mathcal{D})$ of the set $\mathcal{E}$ as follows. A point $w_{0} \in \mathcal{E}$ belongs to $\mathcal{N}$ if there exists a neighborhood $\mathfrak{N}\left(w_{0}\right)$ of $w_{0}$ such that $\mathfrak{N}\left(w_{0}\right)-w_{0}$ contains no point of any essential maximal model continuum of $t\left(w_{0}\right)$.

1.19. If $w_{0} \in \mathcal{E}$, then we have, by definition, in every neighborhood $\mathfrak{N}\left(w_{0}\right)$

(12) This theorem is one of the principal results in the dissertation cited in $\left.{ }^{7}\right)$. 
of $w_{0}$ a Jordan region $\Re$ such that $w_{0} \in \Re^{0}\left({ }^{13}\right)$ and $\Re$ is an indicator region $\left(t\left(w_{0}\right), T\right)$ (cf. $\left.\$ 1.15\right)$. From the definition of an indicator region, it follows that $\mu\left(t\left(w_{0}\right), T, \Re\right) \neq 0$. Generally $\mu\left(t\left(w_{0}\right), T, \Re\right)$ will depend upon the choice of $\Re$. We shall see, however, (cf. \$2.20) that if $w_{0} \in \mathcal{N}$, then there exists a neighborhood $\mathfrak{R}_{0}\left(w_{0}\right)$ of $w_{0}$ such that, for every choice of $\Re$ in $\mathfrak{N}_{0}\left(w_{0}\right)$ (as described above), the quantity $\mu\left(t\left(w_{0}\right), T, \Re\right)$ has one and the same value, which we shall denote by $j\left(w_{0}, T\right)$, and which we shall call the essential local index. For $w_{0}$ not in $\mathcal{N}$, we set $j\left(w_{0}, T\right)=0$. By definition then, we have

$$
\begin{aligned}
& j(w, T)=0 \quad \text { if } \quad w \in \mathcal{D}-\mathcal{N}, \\
& j(w, T) \neq 0 \text { if } w \in \mathcal{N} \text {. }
\end{aligned}
$$

1.20. A fundamental fact about the essential local index $j(w, T)$ is expressed in the theorem: the set $\mathcal{N}_{*}=\mathcal{N}_{*}(T, \mathcal{D})$ of those points $w$ where $|j(w, T)|>1$ is always denumerable $\left({ }^{14}\right)$. It is interesting to observe that the proof of this theorem ( $c f . \$ \$ 2.31-2.40$ ) involves an argument whose scope seems to be restricted to the plane - namely, the use of transformations of the form $z=w^{1 / k}$, where $k$ is a positive integer. Otherwise it seems to us that our theory applies equally well in $n$-dimensional Euclidean space. It would seem that a complete generalization of this theory to $n$-space may necessitate very fascinating geometrical investigations.

1.21. In $\$ \$ 1.8-1.20$ we described the topological facts needed in our work. We turn now to its purely metrical aspects. Given a bounded continuous transformation $T$ defined on a bounded domain $\mathcal{D}$ in the $w$-plane (cf. $\$ 1.13$ ), let us choose a point set $B$ in the $w$-plane (not necessarily contained in $\mathcal{D}$ ), which we shall term the base set. Concerning $\mathbb{B}$ we make the following measurability assumptions: (i) the set $B$ is measurable; (ii) for every oriented( ${ }^{15}$ ) rectangle $R$ such that $R^{0} \subset \mathcal{D}\left({ }^{16}\right)$, the set $T\left(R^{0} \cdot \mathrm{B}\right)$ (cf. $\$ 1.8$ ) is a measurable set in the $z$-plane.

1.22. We may now define a function of rectangles $G(R)$, for all oriented rectangles $R$ such that $R^{0} \subset \mathcal{D}$, as follows $\left({ }^{17}\right)$ :

$$
G(R)=\left|T\left(R^{n} \cdot \mathcal{B}\right)\right| \text {. }
$$

As a matter of fact, $G$ depends upon $R, T$ and $\mathcal{B}$, and should accordingly be denoted by $G(R, T, \mathcal{B})$, but we shall use the notation $G(R)$ unless more explicit notation is needed.

1.23. We shall say that $T$ is of bounded variation, with respect to the base set $\mathbb{B}$, in $\mathcal{D}$-briefly, B.V. $B$ in $\mathcal{D}$-if there exists a finite constant $M$ such that

$$
\sum G\left(s_{i}\right)<M
$$

(13) $\Re^{0}$ denotes the set of interior points of $\Re$.

(14) This theorem is a substantial generalization of a theorem in Rado [5, pp. 199-200].

$\left({ }^{15}\right)$ See $\left.{ }^{4}\right)$.

(16) See $\left(^{13}\right)$.

(17) See $\left({ }^{3}\right)$. 
for every finite sequence of nonoverlapping, closed, oriented squares $s_{i}$ such that $s_{i}^{0} \subset \mathcal{D}$. We shall say that $T$ is absolutely continuous, with respect to the base set $B$ in $\mathcal{D}$-briefly, A.C. $B$ in $\mathcal{D}$-if, for every $\epsilon>0$, there exists an $\eta=\eta(\epsilon)>0$, such that

$$
\sum G\left(s_{i}\right)<\epsilon
$$

for every finite sequence of nonoverlapping, closed, oriented squares $s_{i}$ such that $s_{i}^{0} \subset \mathcal{D}$ and

$$
\sum\left|s_{i}\right|<\eta \text {. }
$$

1.24. In the special case when the base set $B$ is the domain $\mathcal{D}$ itself, these concepts become (essentially) identical to those of Banach (cf. \$1.2). Clearly, if $B$ is a proper subset of $\mathcal{D}$, then our definitions generally require less than those of Banach. It is a matter of fundamental importance for our work that every base set $B$, satisfying the measurability conditions stated in $\$ 1.21$, gives rise to a theory as complete as that of Banach (cf. Chapter 3). We proceed to state the fundamental facts of this general theory.

1.25. Using the measurability assumptions set forth in $\$ 1.21$, one sees easily (cf. $\$ 2.28$ ) that the function $N(z, T, \mathcal{D} \cdot \mathbb{B}$ ) (cf. $\$ 1.10$ ) is measurable. We have then the theorem( ${ }^{18}$ ) (cf. $\left.\$ 3.11\right): T$ is B.V. $\mathcal{B}$ in $\mathcal{D}$ if and only if $N(z, T, \mathcal{D} \cdot \mathbb{B})$ is a summable function.

1.26. Given a point $w \in \mathcal{D}$, let us consider a sequence of oriented closed squares $s_{n}$ such that $w \in s_{n}^{0} \subset \mathcal{D}$ and $\lim \left|s_{n}\right|=0$. If, for every such sequence, the quotients $G\left(s_{n}\right) /\left|s_{n}\right|$ converge to a finite limit (which is then necessarily the same for all such sequences), then this limit is called the derivative of $G(R)$ at $w$ and will be denoted by $D(w)$ - or by $D(w, T, \mathcal{B})$ when the need for more explicit notation arises. We have then the theorem (cf. $\S 3.14,3.15$ ): if $T$ is $B . V . \mathbb{B}$ in $\mathcal{D}$, then $D(w)$ exists a.e. $\left({ }^{19}\right)$ in $\mathcal{D}$, is measurable and summable in $\mathcal{D}$, and we have, for every open set $O \subset \mathcal{D}$, the inequality $\left({ }^{20}\right)$

$$
\iint_{0} D(w, T, \mathscr{B}) \leqq \iint N(z, T, O \cdot \mathbb{B})
$$

1.27. If $T$ is B.V. $B$ in $\mathcal{D}$, then we have, in particular,

$$
\iint_{\mathcal{D}} D(w, T, \mathcal{B}) \leqq \iint N(z, T, \mathcal{D} \cdot \mathcal{B}),
$$

(18) This theorem shows that our concept of bounded variation is independent of the choice of the axes. In a similar way, further theorems in this paper can be easily shown to guarantee this invariant character for the entire theory.

(19) The abbreviation "a.e." is used consistently for "almost everywhere"- "except on a set of measure zero."

$\left.{ }^{(20}\right)$ For conciseness, we omit the symbols $d u d v$ and $d x d y$ respectively in writing double integrals. In the $z$-plane, we shall have to integrate solely functions which vanish outside a sufficiently large disk. The range of integration will be then such a disk, and reference to it will be omitted in the formula. 
and we have the theorem (cf. $\$ \$ 3.20,3.21$ ): T is A.C. $\mathcal{B}$ in $\mathcal{D}$ if and only if the sign of equality holds in the preceding relation.

1.28. Now let $H(z)$ be a finite-valued, measurable function in the $z$-plane. We cannot generally assert that $H(t(w))$ is measurable in $\mathcal{D}$, but we have the theorem (cf. \$3.18) : if $T$ is B.V. B in $\mathcal{D}$ and if $H(z)$ is a finite-valued, measurable function in the $z$-plane, then $H(t(w)) D(w)$ is measurable in $\mathcal{D}$. (Of course, this product is defined only a.e. in $\mathcal{D}$.) Furthermore (cf. \$3.19), if we are given two such functions $H_{1}(z)$ and $H_{2}(z)$, and if $H_{1}(z)=H_{2}(z)$ a.e. in the $z$-plane, then $H_{1}(t(w)) D(w)=H_{2}(t(w)) D(w)$ a.e. in $\mathcal{D}$.

1.29. Assume that $T$ is A.C. $\mathbb{B}$ in $\mathcal{D}$ (cf. $\$ 1.23$ ), and that $H(z)$ is finitevalued and measurable in the z-plane. For every measurable set $E \subset \mathcal{D}$ we have then the fundamental transformation formula (cf. \$3.24):

$$
\iint_{E} H(t(w)) D(w, T, \mathscr{B})=\iint H(z) N(z, T, E \cdot \mathbb{B}),
$$

provided only that one of the two integrals involved exists. That is, if one of the two integrals exists, then the other one exists also, and the two are equal.

1.30. We shall combine presently the topological facts (cf. \$\$1.8-1.20) and the metrical facts (cf. \$\$1.21-1.29) at our disposal. Given a bounded continuous transformation $T$ defined on a bounded domain $\mathcal{D}$ in the w-plane (cf. $\$ 1.13$ ), we select the essential set $\mathcal{E}$ defined in $\$ 1.18$ as the base set (cf. $\$ 1.21$ ) in the preceding metrical theory. We shall verify (cf. $\$ \$ 2.22-2.29$ ) that $\varepsilon$ satisfies the measurability requirements stated in 1.21 . We shall then proceed to study continuous transformations $T$ which are B.V. $\mathcal{E}$ in $\mathcal{D}$ and A.C. $\mathcal{E}$ in $\mathcal{D}$ respectively (cf. $\$ \$ 1.23,1.18$ ). In the proofs (cf. Chapter 4 ), we shall have to consider several auxiliary set functions, together with their derivatives; the derivative $D(w, T, \varepsilon)$ of $G(R, T, \varepsilon)$ (cf. $\$ 1.22)$ will be denoted by $D_{2}(w)$ or by $D_{2}(w, T)$ when more explicit notation is needed. In case $T$ is B.V. $\mathcal{E}$ in $\mathcal{D}$, it follows that $D_{2}(w)$ exists a.e. in $\mathcal{D}$ and is summable in $\mathcal{D}$ (cf. $\left.\$ 1.26\right)$. We then define a generalized Jacobian (cf. \$4.3) by the relation

$$
\mathcal{F}(w, T)=j(w, T) D_{2}(w, T)
$$

which we also denote by $\mathcal{F}(w)$. From $\S 1.20$ it follows that $|\mathcal{F}(w)| \leqq D_{2}(w)$ a.e. in $\mathcal{D}$; hence $\mathcal{F}(w)$ is also summable in $\mathcal{D}$.

1.31. Given any domain $\mathcal{D}_{*} \subset \mathcal{D}$, we define an essential index function $\nu\left(z, T, \mathcal{D}_{*}\right)$ as follows (cf. $\left.\$ \S 1.8,1.10,1.18,1.19\right)$ :

$$
\nu\left(z, T, \mathcal{D}_{*}\right)=\left\{\begin{array}{l}
\sum_{w} j(w, T) \text { for } w \in \mathcal{E} \cdot \mathscr{D}_{*} \cdot T^{-1}(z), \text { if } z \text { not in } \overline{\mathfrak{S}}\left(\infty, T, \mathcal{E} \cdot \mathscr{D}_{*}\right), \\
0 \text { if } z \in \overline{\mathfrak{S}}\left(\infty, T, \mathcal{E} \cdot \mathcal{D}_{*}\right) .
\end{array}\right.
$$

We shall show (cf. $\$ 2.30)$ that $\nu\left(z, T, D_{*}\right)$ is a summable function provided that $T$ is B.V. $\mathcal{E}$ in $\mathcal{D}_{*}$. 
1.32. Given a bounded domain $\mathcal{D}$ in the w-plane, our fundamental class of transformations on $\mathcal{D}$ will be denoted by $K_{1}(\mathcal{D})$ and will consist of all those bounded continuous transformations defined on $\mathcal{D}$ (cf. \$1.13) which are A.C. $\mathcal{E}_{\text {in }}$ $\mathcal{D}\left({ }^{21}\right)$ (cf. §1.23). By the general metric theory described above (cf. \$1.29), we have then, for every finite-valued, measurable function $H(z)$ and for every transformation $T \in K_{1}(\mathcal{D})$ the metrical transformation formula

$$
\iint_{\mathcal{D}} H(t(w)) D_{2}(w, T)=\iint H(z) N(z, T, \mathcal{D} \cdot \mathcal{E}),
$$

as soon as one of these integrals exists. We shall establish (cf. §4.9), for every finite-valued, measurable function $H(z)$ and for every transformation $T \in K_{1}(\mathcal{D})$ the topological transformation formula (cf. \$1.31)

$$
\iint_{\mathcal{D}} H(t(w)) \mathcal{F}(w, T)=\iint H(z) \nu(z, T, \mathcal{D}),
$$

provided that the integral on the left exists. That is, if the integral on the left exists, then the integral on the right exists, and the integrals are equal. The converse is generally false (see Radó [5, p. 214]).

1.33. The preceding formulas are of extreme generality. In particular, the topological transformation formula of $\$ 1.32$ implies all the results on the transformation of double integrals in the literature of which we are aware (cf. Bibliography). As a matter of fact, very special cases of the preceding results are ample for that purpose. We proceed now to describe such special cases for the purpose of applications to be presented on another occasion.

1.34. Given a bounded domain $\mathcal{D}$ in the w-plane, let $K_{2}(\mathcal{D})$ denote the class of all transformations $T \in K_{1}(\mathcal{D})$ (cf. $\$ 1.32$ ) for which $N(z, T, \mathcal{E} \cdot \mathcal{D})$ $=\kappa(z, T, \mathcal{D})$ a.e. in the $z$-plane (cf. $\$ \S 1.10,1.13,1.18)$. For transformations $T \in K_{2}(\mathcal{D})$ we shall see (cf. \$4.7) that $|\mathcal{F}(w)|=D_{2}(w)$ a.e. in $\mathcal{D}$ (cf. \$1.30). Next, we shall consider the special case when $\mathcal{D}$ is the interior $\Re^{0}$ of a Jordan region $\Re$ (cf. \$4.9). For this case, we shall derive the following theorem: if $T$ is continuous on the Jordan region $\Re$, if $T \in K_{2}\left(\Re^{0}\right)$, and if the image of the boundary of $\Re$ is a set of measure zero, then, for every finite-valued, measurable function $H(z)$ we have (cf. \$1.14)

$$
\iint_{\mathfrak{R}^{0}} H(t(w)) \mathcal{F}(w)=\iint H(z) \mu(z, T, \Re),
$$

as soon as the integral on the left exists.

1.35. A fundamental property of the class $K_{2}(\mathcal{D})$ is expressed in the following:

(21) That is, $T \in K_{1}(\mathcal{D})$ if $T$ is absolutely continuous in $\mathcal{D}$ with respect to its own essential set $\varepsilon=\varepsilon(T, \mathcal{D})$. 
Closure theorem. Let there be given bounded domains $\mathcal{D}$ and $\mathcal{D}_{n}$ in the w-plane, and continuous transformations ( $c f . \S 1.8)$

$$
T: \quad z=t(w), w \in \mathcal{D}, \quad T_{n}: \quad z=t_{n}(w), w \in \mathcal{D}_{n},
$$

with the following properties: (i) the domains $\mathcal{D}_{n}$ fill up $\mathcal{D}$ from the interior $\left({ }^{22}\right)$; (ii) the generalized Jacobian $\mathcal{F}(w, T)$ exists a.e. in $\mathcal{D}$ and is summable in $\mathcal{D}$; (iii) $T_{n} \in K_{2}\left(\mathcal{D}_{n}\right)$ for $n=1,2, \cdots$; (iv) for every closed set $F \subset \mathcal{D}$ we have $\lim \rho\left(T_{n}, T ; F\right)=0(c f . \S 1.9)$, and

$$
\lim \iint_{F}\left|\mathcal{F}(w, T)-\mathcal{F}\left(w, T_{n}\right)\right|=0 .
$$

Then $T \in K_{2}(\mathcal{D})(c f . \S 4.11)$.

1.36. Next, we have the following theorem (cf. $\$ 4.14)$ : in a Jordan region $\Re$ let there be given continuous transformations $T$ and $T_{n}$ with the following properties: (i) $T \in K_{2}\left(\Re^{0}\right), T_{n} \in K_{2}\left(\Re^{0}\right)$; (ii) $\lim \rho\left(T_{n}, T\right.$; $\left.\Re\right)=0$; (iii) the image of the boundary of $\Re$ under $T$ is a set of measure zero; (iv) we have

$$
\lim \iint_{\Re^{0}}\left|\mathcal{F}(w, T)-\mathcal{F}\left(w, T_{n}\right)\right|=0 .
$$

Then (cf. $\$ 1.14)$

$$
\lim \iint\left|\mu(z, T, \Re)-\mu\left(z, T_{n}, \Re\right)\right|=0 .
$$

That is, strong convergence of the generalized Jacobians (cf. \$1.30) implies strong convergence of the index functions $\mu$ (cf. \$1.14).

1.37. We now write the transformation $T$ in the form (cf. §1.8)

$$
T: \quad x=x(u, v), \quad y=y(u, v), \quad(u, v) \in \mathcal{D},
$$

and denote by $K_{3}(\mathcal{D})$ the class of all transformations $T \in K_{2}(\mathcal{D})$ (cf. $\$ 1.34$ ) for which the ordinary Jacobian $J(w, T)=J(u, v, T)=x_{u} y_{v}-x_{v} y_{u}$ exists a.e. in $\mathcal{D}$.

Note that this is the first time that we refer to the ordinary Jacobian, either in the theorems or in the proofs.

1.38. We have the important theorem (cf. §5.5): if $T \in K_{3}(\mathcal{D})$, then $J(w, T)=\mathcal{F}(w, T)$ a.e. in $\mathcal{D}$. This theorem permits us to replace $\mathcal{F}(w, T)$ by $J(w, T)$ in the transformation formulas in $\$ 1.32$ and $\$ 1.34$. In this fashion we obtain formulas which include all similar results in the literature of which we are aware. We now list some theorems which account for these results.

1.39. Suppose that the functions $x(u, v), y(u, v)$ defining $T$ (cf. $\S 1.37$ ) satisfy a Lipschitz condition in $\mathcal{D}$ in the following restricted sense : there exists

(22) The domains $\mathcal{D}_{n}$ are said to fill up $\mathcal{D}$ from the interior if (i) $\mathcal{D}_{n} \subset \mathcal{D}$ and (ii) for every closed set $F \subset \mathcal{D}$, there exists an $n_{0}=n_{0}(F)$ such that $F \subset \mathcal{D}_{n}$ for all $n>n_{0}$. 
a finite constant $L$ such that, if $\left(u_{1}, v_{1}\right),\left(u_{2}, v_{2}\right)$ are any two points in $\mathscr{D}$ for which the line segment joining them is contained in $\mathcal{D}$, then

$$
\left.\mid \begin{array}{l}
x\left(u_{2}, v_{2}\right)-x\left(u_{1}, v_{1}\right) \mid \\
y\left(u_{2}, v_{2}\right)-y\left(u_{1}, v_{1}\right) \mid
\end{array}\right\} \leqq L\left[\left(u_{2}-u_{1}\right)^{2}+\left(v_{2}-v_{1}\right)^{2}\right]^{1 / 2} .
$$

Then $T \in K_{3}(D)$ (cf. $\S 5.8$ ).

1.40. Suppose that the partial derivatives $x_{u}, x_{v}, y_{u}, y_{v}$ exist and are continuous in $\mathcal{D}$ and that the ordinary Jacobian $J(w, T)$ is summable in $\mathscr{D}$. Then $T \in K_{3}(\mathcal{D})$ (cf. §5.9).

1.41. For the class $K_{3}(\mathcal{D})$ we have the following:

Closure theorem. Let there be given bounded domains $\mathcal{D}$ and $\mathcal{D}_{n}$ in the $u v$-plane, and continuous transformations ( $c f . \S 1.8$ )

$$
\begin{aligned}
& T: x=x(u, v), \quad y=y(u, v), \quad(u, v) \in \mathcal{D}, \\
& T_{n}: \quad x=x_{n}(u, v), \quad y=y_{n}(u, v), \quad(u, v) \in \mathcal{D}_{n},
\end{aligned}
$$

with the following properties: (i) the domains $\mathcal{D}_{n}$ fill up $\mathcal{D}$ from the interior $\left({ }^{23}\right)$; (ii) the ordinary Jacobian $J(w, T)$ exists a.e. in $\mathcal{D}$ and is summable in $\mathcal{D}$; (iii) $T_{n} \in K_{3}\left(\mathcal{D}_{n}\right)$ for $n=1,2, \cdots$; (iv) for every closed set $F \subset \mathcal{D}$ we have $\lim \rho\left(T_{n}, T ; F\right)=0$ (cf. §1.9), and

$$
\lim \iint_{F}\left|J(w, T)-J\left(w, T_{n}\right)\right|=0 .
$$

Then $T \in K_{3}(\mathcal{D})(c f . \S 5.7)$.

1.42. The reader will note that the ordinary Jacobian $J(u, v)=x_{u} y_{v}-x_{v} y_{u}$ appears only in the last few sections, after the general theory has been completed. As far as our theory is concerned, the ordinary Jacobian is entirely irrelevant, and we consider it solely for the purpose of applications to previous literature. This situation leads quite naturally to the question as to whether the ordinary Jacobian is really an essential concept in formulating and studying fundamental problems involving double integrals in analysis and in geometry.

\section{Chapter II. Topological foundations}

2.1. In this chapter we shall give concise proofs of the geometrical facts in this theory. Most of these facts have been stated in $\$ \$ 1.8-1.20$. In order to verify them, however, we shall find it necessary to use many auxiliary results; for the proofs of these, we refer the reader to the literature, unless we are aware of no simple published proofs.

2.2. Let $\Re$ be a Jordan region in the $w$-plane (cf. $\S 1.11$ ), and let $T$ be a

${ }^{(23)}$ See $\left({ }^{22}\right)$. 
continuous transformation defined on $\Re$ (cf. 1.8). We state two properties of the kernel of order $k$ (cf. $\$ 1.11)$ needed in the sequel.

LEMmA 1. Every kernel $\bar{\Re}(k, T, \Re)$ of finite order is an open set $\left({ }^{24}\right)$.

Proof ${ }^{(25)}$. If $\bar{\Omega}$ is empty, the lemma is trivial. So suppose $z \in \bar{\Re}$; we assert that every point $z_{*}$ satisfying $\left|z_{*}-z\right|<\epsilon=\epsilon(k, z, T, \Re)$ (cf. $\left.\$ 1.11\right)$ is also in $\bar{\Omega}$. Set $\lambda=z-z_{*}, \epsilon_{*}=\epsilon-|\lambda|$; clearly $\epsilon_{*}>0$. If $T_{*}$ be any continuous transformation defined on $\Re$ and satisfying $\rho\left(T_{*}, T ; \Re\right)<\epsilon_{*}$ (cf. 1.9), then clearly the transformation $T^{*}$ defined by $t^{*}(w)=t_{*}(w)+\lambda, w \in \Re$, is continuous on $\Re$ and satisfies $\rho\left(T^{*}, T ; \Re\right)<\epsilon$; consequently $N\left(z, T^{*}, \Re\right) \geqq k$ (cf. $\left.\S 1.11\right)$. But obviously $N\left(z_{*}, T_{*}, \Re\right)=N\left(z, T^{*}, \Re\right) \geqq k$; thus $z^{*} \in \bar{\Re}$ by definition.

LEMMA 2. If $\left\{T_{n}\right\}$ be a sequence of continuous transformations defined on $\Re$ and satisfying $\lim \rho\left(T_{n}, T ; \Re\right)=0$, then $\lim \inf \bar{\Re}\left(k, T_{n}, \Re\right) \supset \bar{\Omega}(k, T, \Re)$, provided $k$ is finite.

The proof is obvious (see Radó [5, p. 205]).

2.3. We pause to introduce some definitions and notations useful in the sequel. Given a function $f(w)$ defined on some set in the w-plane, and any set $E$ on which it is defined, we denote by $M(f, E)$ the least upper bound of $|f(w)|$ for $w \in E$; we denote by $O(f, E)$ the least upper bound of $\left|f\left(w^{\prime}\right)-f\left(w^{\prime \prime}\right)\right|$ for $w^{\prime}, w^{\prime \prime} \in E$. Obviously $O(f, E) \leqq 2 M(f, E)$. If $\delta$ be any positive number, we denote by $O(\delta, f, E)$ the least upper bound of $\left|f\left(w^{\prime}\right)-f\left(w^{\prime \prime}\right)\right|$ for $w^{\prime}$, w' $\in E$, $\left|w^{\prime}-w^{\prime \prime}\right| \leqq \delta$. If $f(w)$ is continuous and $E$ is a bounded closed set, then $O(\delta, f, E)$ converges to zero with $\delta$.

2.4. In $\$ 1.12$ we observed that $\kappa(z, T, \Re) \leqq N(z, T, \Re)$. However, we shall have use for the stronger result contained in the

LEмMA. $\kappa(z, T, \Re) \leqq N\left(z, T, \Re^{0}\right)\left({ }^{26}\right)$.

Proof. Clearly it is sufficient to show that, if $N\left(z, T, \Re^{0}\right)=k<+\infty$, then, for every $\epsilon>0$, there exists a continuous transformation $T_{\epsilon}$ defined on $\Re$ and satisfying $\rho\left(T_{\epsilon}, T ; \Re\right)<\epsilon$ and $N\left(z, T_{\epsilon}, \Re\right) \leqq k$. Let $\bar{\Re}$ be a Jordan region (cf. $\S 1.11)$ in a $\bar{w}$-plane having the same connectivity as $\Re$, each of whose boundary curves is a circle. Map $\Re$ onto $\bar{\Re}$ by a biunique and continuous transformation given by $\bar{w}=f(w), w \in \Re$ (see Kerékjártó [1]). The inverse transformation given by $w=f^{-1}(\bar{w}), w \bar{w} \in \bar{\Re}$, is also biunique and continuous, and maps $\bar{\Re}$ onto $\Re$. Define a continuous transformation $\bar{T}$ from $\bar{\Re}$ to the $z$-plane by the relation $z=\bar{t}(\bar{w})=t\left(f^{-1}(\bar{w})\right), \bar{w} \in \bar{\Re}$ (cf. $\left.\S 1.8\right)$. Let $\delta$ be a positive number such that $O(\delta, \bar{t}, \bar{\Re})<\epsilon(\mathrm{cf} . \S 2.3)$. If $\bar{C}$ denotes a typical boundary circle of $\bar{\Re}$, choose in $\bar{\Re}^{0}$ a concentric circle $\bar{C}_{\epsilon}$ at a distance less than $\delta$ from $\bar{C}$ so that the

(24) Rado (see Radó [5, p. 203]) proved the weaker result that every kernel of finite order is an $F_{\sigma}$.

(25) For brevity, we write $\bar{\Omega}$ for $\bar{\Omega}(k, T, \Re)$ if the meaning is clear from the context.

${ }^{(26)}$ See ${ }^{(13)}$. 
circles $\bar{C}_{\epsilon}$ bound a Jordan region $\bar{\Re}_{\epsilon}$ in $\bar{\Re}^{0}$ having the same connectivity as $\bar{\Re}$ and for which $N\left(z, \bar{T}, \bar{\Re}_{\epsilon}\right)=N\left(z, \bar{T}, \bar{\Re}_{\epsilon}^{0}\right) \leqq k$. Define a continuous transformation $\bar{T}_{\epsilon}$ from $\Re$ to the $z$-plane by the relations

$$
\bar{T}_{\epsilon}: \quad z=\bar{t}_{\epsilon}(\bar{w})=\left\{\begin{array}{l}
\bar{t}(\bar{w}), \quad \bar{w} \in \overline{\Re_{\epsilon}} ; \\
\bar{t}\left(\bar{w}_{*}\right), \bar{w}_{*} \in \bar{\Re}-\bar{\Re}_{\epsilon},
\end{array}\right.
$$

where $\bar{w}_{*}$ is the closest point to $\bar{w}$ on a boundary curve of $\bar{\Re}_{\epsilon}$. Clearly $\rho\left(\bar{T}_{\epsilon}, \bar{T} ; \bar{\Re}\right)<\epsilon$ and $N\left(z, \bar{T}_{\epsilon}, \bar{\Re}\right)=N\left(z, \bar{T}, \bar{\Re}_{\epsilon}^{0}\right) \leqq k$. Finally, set $T_{\epsilon}: z=t_{\epsilon}(w)$ $=\bar{t}_{\epsilon}(f(w)), w \in \Re$. Clearly the transformation $T_{\epsilon}$ thus defined (cf. $\$ 1.8$ ) has the required properties.

2.5. As a corollary to the results in $\$ 2.2$ and $\$ 2.4$ and to the definition of the essential multiplicity (cf. $\$ 1.12$ ), it follows that $\kappa(z, T, \Re)$ is a lower semicontinuous function of $z$ and of $T\left({ }^{27}\right)$, as asserted in $\$ 1.12$.

2.6. We turn now to establish the important

TheOREM. $\kappa(z, T, \Re)$ is equal to the number of distinct essential maximal model continua of $z$ under $T$ in $\Re^{0}(c f . \$ 1.16)$.

The proof of this theorem will follow (cf. \$2.17) from a series of simple facts which we presently state and verify. The reader will easily see that this theorem accounts for all the assertions made in $\$ 1.13$ and for the fundamental characterization theorem for the essential multiplicity function $\kappa(z, T, \mathcal{D})$ for a domain $\mathcal{D}$ which is stated in $\$ 1.17$.

2.7. To approach the proof of the theorem stated in $\$ 2.6$ (and for later purposes also), we need the following facts concerning the function $\mu(z, T, \Re)$ defined in $\$ 1.14$. Given a point $z_{0}$ in the $z$-plane, define $\epsilon\left(z_{0}, T, \Re\right)$ to be the minimum of $\left|t(w)-z_{0}\right|$ for $w \in \Re-\Re^{0}\left({ }^{28}\right)$. Clearly $\epsilon\left(z_{0}, T, \Re\right)=0$ if and only if $z_{0} \in T\left(\Re-\Re^{0}\right)$ (cf. $\$ 1.8$ ).

Lemma 1. Consider the continuous transformations (cf. \$1.8)

$$
T_{i}: \quad z=t_{i}(w), \quad w \in \Re, i=0,1, \cdots, m .
$$

Suppose that, for points $z_{0}, z_{1}, \cdots, z_{m}$, it is true that $\epsilon\left(z_{i}, T, \Re\right)>0$ for $i=1, \cdots, m$, and

Then

$$
t_{0}(w)-z_{0}=\prod_{i=1}^{m}\left[t_{i}(w)-z_{i}\right], \quad w \in \Re
$$

$$
\mu\left(z_{0}, T_{0}, \Re\right)=\sum_{i=1}^{m} \mu\left(z_{i}, T_{i}, \Re\right)
$$

(27) A sequence of continuous transformations $T_{n}$ defined on $\Re$ is said to converge to $T$ on $\Re$ if $\lim \rho\left(T_{n}, T ; \Re\right)=0$ (cf. $\$ 1.9$ ).

(28) $\Re-\Re^{0}$ is the set of boundary points of $\Re$. See $\left(^{8}\right),\left({ }^{13}\right)$. 
This lemma is a ready consequence of the properties of the topological index (cf. §1.14).

Lemma 2. Assume that $\epsilon(z, T, \Re)>0$. ( $\alpha$ ) If $z_{*}$ be any point in the z-plane satisfying $\left|z_{*}-z\right|<\epsilon(z, T, \Re)$, then $\mu\left(z_{*}, T, \Re\right)=\mu(z, T, \Re)$. ( $\left.\beta\right)$ If $T_{*}$ be any continuous transformation defined on $\Re$ and satisfying $\rho\left(T_{*}, T ; \Re-\Re^{0}\right)$ $<\epsilon(z, T, \Re)$, then $\mu\left(z, T_{*}, \Re\right)=\mu(z, T, \Re)$.

This lemma is an immediate consequence of the theorem of Rouché( $\left.{ }^{29}\right)$.

Lemma 3. $\mu(z, T, \Re)=0$ for $z$ not $\in T(\Re)$ (see Radó [5, p. 196] also for further references).

LemmA 4. Assume that $\Re$ is simply connected. Let $z_{0}$ be a point of the z-plane for which $\epsilon\left(z_{0}, T, \Re\right)>0$ and $\mu\left(z_{0}, T, \Re\right)=0$. Then there exists a function $f(w)$ having the following properties: (i) $f(w)$ is defined and continuous on $\Re$; (ii) $f(w)=t(w)$ for $w \in \Re-\Re^{0}$; (iii) $M\left(f(w)-z_{0}, \Re\right)=M\left(t(w)-z_{0}, \Re-\Re^{0}\right)$; (iv) $f(w) \neq z_{0}$ for $w \in \Re$ (see Radó [5, p. 196] also for further references).

Lemma 5. Let $\Re_{1}, \cdots, \Re_{m}$ be any finite number of nonoverlapping Jordan regions in $\Re-\Re_{0}$. Given a point $z_{0}$ in the $z$-plane, let $\zeta=\zeta\left(z_{0}, T,\left\{\Re_{i}\right\}_{0}^{m}\right)$ denote the greatest lower bound of $\left|t(w)-z_{0}\right|$ for $w \in \Re_{0}-\sum_{i=1}^{m} \Re_{i}^{0}$. If $z_{0}$ be any point for which $\zeta>0$, then $\mu\left(z_{0}, T, \Re_{0}\right)=\sum_{i=1}^{m} \mu\left(z_{0}, T, \Re_{i}\right)$.

Proof. Clearly $\epsilon\left(z_{0}, T, \Re_{i}\right) \geqq \zeta>0$ for $i=0,1, \cdots, m$. Let $T_{*}$ be any continuous transformation defined on $\Re$, and such that $\rho\left(T_{*}, T ; \Re\right)<\zeta$ and $z_{0}$ not $\in \overline{\mathfrak{S}}\left(\infty, T_{*}, \Re\right)(\mathrm{cf} . \S 1.10)\left({ }^{30}\right)$. From Lemma 2 , it follows that $\mu\left(z_{0}, T_{*}, \Re_{i}\right)$ $=\mu\left(z_{0}, T, \Re_{i}\right)$ for $i=0,1, \cdots, m$. Since $z_{0}$ not $\in \bar{S}\left(\infty, T_{*}, \Re\right)$, the sets $T_{*}^{-1}\left(z_{0}\right) \cdot \Re_{i}^{0}$ are finite for $i=0,1, \cdots, m$ (cf. $\left.\S \S 1.8,1.10\right)$. We shall discuss the general case when none of these sets are empty, leaving special cases for the reader. If $w_{i 1}, \cdots, w_{i j_{i}}$ denote the points of $T_{*}^{-1}\left(z_{0}\right) \cdot \Re_{i}^{0}$ for $i=1, \cdots, m$, then clearly the points in the set $T_{*}^{-1}\left(z_{0}\right) \cdot \Re_{0}^{0}$ consist of

$$
w_{11}, \cdots, w_{1 j_{1}}, \cdots, w_{m 1}, \cdots, w_{m j_{m}} \text {. }
$$

Choose $j_{i}$ mutually exclusive simply connected Jordan regions $\Re_{i j}$ such that $w_{i j} \in \Re_{i j}^{0}$ for $j=1, \cdots, j_{i}, i=1, \cdots, m$. As a consequence of Lemma 3 , and of the assumption $\zeta>0$, it follows that $\mu\left(z_{0}, T_{*}, \Re_{i}\right)=\sum_{j=1}^{j_{i}} \mu\left(z_{0}, T_{*}, \Re_{i j}\right)$ for $i=1,2, \cdots, m$, and

$\left.{ }^{29}\right)$ We mean the purely topological statement of the theorem of Rouché as given, for example, in Szilárd [1, p. 655].

(30) The existence of such a transformation as $T_{*}$ may be inferred as follows. Since every Jordan region is topologically equivalent to a polygonal region of the same connectivity, we may assume that $\Re$ is a polygonal region - that is, a Jordan region each of whose boundary curves is a simple polygon. Given $\zeta>0$ one may choose a triangulation of $\Re$ so fine that one may define upon $\Re$ a continuous transformation $T_{\zeta}$ which is linear and biunique in each triangle of the triangulation, and for which $\rho\left(T_{\zeta}, T ; \Re\right)<\zeta$. Clearly $\widetilde{\Phi}\left(\infty, T_{\zeta}, \Re\right)$ is empty; thus $T_{\zeta}$ has the desired properties. 


$$
\mu\left(z_{0}, T_{*}, \Re_{0}\right)=\sum_{i=1}^{m} \sum_{j=1}^{j_{i}} \mu\left(z_{0}, T_{*}, \Re_{i j}\right)=\sum_{i=1}^{m} \mu\left(z_{0}, T_{*}, \Re_{i}\right) .
$$

In view of preceding equalities, the lemma now follows.

2.8. Using Lemma 2 in $\$ 2.7$ and the definition of an indicator system $(z, T)$ (cf. $\$ 1.15$ ), the reader will easily verify the

Lemma. If $\mathfrak{S}$ be any indicator system $(z, T)$ of order $k$, then $\epsilon(z, T, \mathfrak{S})=$ the minimum of $\epsilon\left(z, T, \Re_{*}\right)$ for $\Re_{*} \in \mathfrak{S}$, is positive. $(\alpha)$ For every point $z_{*}$ satisfying $\left|z_{*}-z\right|<\epsilon(z, T, \mathfrak{S})$, it is true that $\mathfrak{S}$ is also an indicator system $\left(z_{*}, T\right)$ of order $k$, and $\mu\left(z_{*}, T, \Re_{*}\right)=\mu\left(z, T, \Re_{*}\right)$ for every $\Re_{*} \in \Im$. $(\beta)$ For every continuous

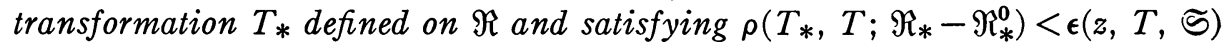
for every $\Re_{*} \in \mathfrak{S}$, it is true that $\mathfrak{S}$ is also an indicator system $\left(z, T_{*}\right)$ of order $k$, and $\mu\left(z, T_{*}, \Re_{*}\right)=\mu\left(z, T, \Re_{*}\right)$ for every $\Re_{*} \in \subseteq$.

2.9. Let $z_{0}$ be any point in the $z$-plane. The least upper bound of the orders of all indicator systems $\left(z_{0}, T\right)$ in $\Re^{0}$ will be denoted by $\psi\left(z_{0}, T, \Re^{0}\right)$. It follows from Lemma 3 in $\$ 2.7$ that every indicator region $\left(z_{0}, T\right)$ contains (in its interior) at least one point of $T^{-1}\left(z_{0}\right)$ (cf. $\left.\$ 1.15\right)$. Consequently, in view of the definitions of $\kappa\left(z_{0}, T, \Re\right)$ (cf. $\$ 1.12$ ), and of an indicator system $\left(z_{0}, T\right.$ ) (cf. $\S 1.15)$, it follows from the lemma in $\S 2.8$ that $\psi\left(z_{0}, T, \Re^{0}\right) \leqq \kappa\left(z_{0}, T, \Re\right)$. Further, if $\phi\left(z_{0}, T, \Re^{0}\right)$ denotes the number of essential maximal model continua of $z_{0}$ under $T$ in $\Re^{0}$ (cf. §1.16), it is clear that $\phi\left(z_{0}, T, \Re^{0}\right) \leqq \psi\left(z_{0}, T, \Re^{0}\right)$.

2.10. Assume, for the moment, that $z$ not $\in \overline{\mathfrak{S}}\left(\infty, T, \Re^{0}\right)(\mathrm{cf} . \S 1.10)$. Then the point set $T^{-1}(z) \cdot \Re^{0}$ is finite; consequently, if $w \in T^{-1}(z) \cdot \Re^{0}$, then there exists a neighborhood $\mathfrak{N}_{0}(w)$ of $w$ containing no further points of $T^{-1}(z)$. Now, for every Jordan region $\Re_{*}$ in $\Re_{0}(w)$ and containing $w$ in its interior, it follows by Lemma 3 in $\S 2.7$ that $\mu\left(t(w), T, \Re_{*}\right)$ has the same value. If $\mu\left(t(w), T, \Re_{*}\right) \neq 0$, then clearly $w$ constitutes an essential maximal model continuum of $z=t(w)$ belonging to the set $\mathcal{N}\left(T, \Re^{0}\right)$ (cf. $\S 1.18$ ). Thus the assertion made in $\$ 1.19$ regarding points of $\mathcal{N}$ is now verified for all points $w \in \mathcal{N}$ for which $t(w)$ is not $\in \overline{\mathfrak{S}}\left(\infty, T, \Re^{0}\right)$. Further, it is obvious that $\phi\left(z, T, \Re^{0}\right)=N(z, T, \mathcal{N})$ in this case (cf. §2.9). But, moreover, we have the

Lemma. If $z$ not $\in \overline{\mathfrak{S}}\left(\infty, T, \Re^{0}\right)$, then $\kappa(z, T, \Re)=N(z, T, \mathcal{N})\left({ }^{31}\right)$.

Proof. A point $z_{0}$ not $\in \bar{\Phi}\left(\infty, T, \Re^{0}\right)$ has a finite number of models under $T$ in $\Re^{0}$; denote the points of $T^{-1}\left(z_{0}\right)$ in $\mathcal{N}$ by $w_{1}, \cdots, w_{k}$; denote the points of $T^{-1}\left(z_{0}\right)$ in $\Re^{0}-\mathcal{N}$ by $w_{k+1}, \cdots, w_{l}$. We shall first show that $\kappa \geqq k\left({ }^{32}\right)$. Evidently one may choose $k$ mutually exclusive (simply connected) Jordan regions $\Re_{j} \subset \Re^{0}$ so that $w_{j} \in \Re_{j}^{0}$ and $T^{-1}\left(z_{0}\right) \cdot \Re_{j}=w_{j}$ for $j=1, \cdots, k$. Since

(31) Radó (see Radó [5, p. 204]) used the same methods in proving a slightly less general result.

(32) For brevity, we write $\kappa$ for $\kappa(z, T, \Re)$ where no misunderstanding is possible. 
clearly $\mu\left(z_{0}, T, \Re_{j}\right)=j\left(w_{j}, T\right) \neq 0$ for $j=1, \cdots, k$ (cf. $\$ 1.19$ ), it follows by $\$ 1.15$ that the $k$ Jordan regions $\Re_{j}$ constitute an indicator system $\left(z_{0}, T\right)$ of order $k$, hence $\kappa \geqq k$ (cf. $\$ 2.9)$. We shall next prove that $\kappa \leqq k$. In view of the lemma in $\$ 2.4$, it is sufficient to exhibit, for every $\epsilon>0$, a continuous transformation $T_{\epsilon}$ defined on $\Re$ for which $\rho\left(T_{\epsilon}, T ; \Re\right)<\epsilon$ and $N\left(z_{0}, T_{\epsilon}, \Re^{0}\right) \leqq k$. One may obviously choose $l-k$ mutually exclusive simply connected Jordan regions $\Re_{j} \subset \Re^{0}$ so that $w_{j} \in \Re_{j}^{0}, T^{-1}\left(z_{0}\right) \cdot \Re_{j}=w_{j}$, and $M\left(t(w)-z_{0}, \Re_{j}\right)<\epsilon / 2$ for $j=k+1, \cdots, l$. Since $w_{j} \in \Re^{0}-\mathcal{N}$, it follows that $\mu\left(z_{0}, T, \Re_{j}\right)=j\left(w_{j}, T\right)=0$ for $j=k+1, \cdots, l$ (cf. $\$ 1.19$ ). Thus by Lemma 4 in $\$ 2.7$, there exist $l-k$ functions $f_{j}(w)$ for $j=k+1, \cdots, l$ with the following properties: (i) $f_{j}(w)$ is defined and continuous on $\Re_{j}$; (ii) $f_{j}(w)=t(w)$ for $w \in \Re_{j}-\Re_{j}^{0}$; (iii) $M\left(f_{j}(w)-z_{0}, \Re_{j}\right.$ ) $<\epsilon / 2$; (iv) $f_{j}\left(w_{0}\right) \neq z_{0}$ for $w \in \Re_{j}$. Define $T_{\epsilon}$ by the relations

$$
T_{\epsilon}: \quad z=t_{\epsilon}(w)=\left\{\begin{array}{l}
t(w) \text { if } w \in \Re-\sum_{j=k+1}^{l} \Re_{j}, \\
f_{j}(w) \text { if } w \in \Re_{j}, \quad j=k+1, \cdots, l .
\end{array}\right.
$$

Clearly $T_{\epsilon}$ is a continuous transformation defined on $\Re, \rho\left(T_{\epsilon}, T ; \Re\right)<\epsilon$, and $N\left(z_{0}, T_{\epsilon}, \Re^{0}\right)=k$. Combining preceding inequalities, we have $\kappa\left(z_{0}, T, \Re\right)=k$ $=N\left(z_{0}, T, \mathcal{N}\right)$, as asserted.

2.11. Surveying the remarks in $\$ \S 2.9,2.10$ and the lemma in $\$ 2.10$, we observe that the theorem stated in $\$ 2.6$ is now established in the special case when $z$ not $\in \overline{\mathfrak{S}}\left(\infty, T, \Re^{0}\right)$.

2.12. Given a continuous transformation $T$ defined on a Jordan region $\Re$ (cf. $\$ 1.8)$. Let $\tau$ be any positive number, and $z_{0}$ any point of the $z$-plane; denote by $G=G\left(\tau, z_{0}, T, \Re\right)$ the set of all points $w \in \Re^{0}$ for which $\left|t(w)-z_{0}\right|<\tau$. Clearly $T^{-1}\left(z_{0}\right) \subset G$. Since $G$ is an open set, it is the sum of at most a denumerable number of mutually exclusive components, which we shall denote generically by $g=g\left(\tau, z_{0}, T, \Re\right)$. Each set $g$ is a connected open set; denote by $\bar{g}$ the set $g$ plus all its boundary points. Clearly $\left|t(w)-z_{0}\right|=\tau$ for $w \in(\bar{g}-g) \cdot \Re^{0}$; thus, if $w_{0}$ be a point of $\bar{g}-g$ for which $\left|t\left(w_{0}\right)-z_{0}\right|<\tau$, it follows that $w_{0} \in \Re-\Re^{0}$.

2.13. Lemma 1. Assume that $z_{0}$ not $\in \bar{S}\left(\infty, T, \Re^{0}\right)$. If $g$ be any component of $G=G\left(\tau, z_{0}, T, \Re\right)$ for which $\left|t(w)-z_{0}\right|=\tau$ for $w \in \bar{g}-g$ and $N\left(z_{0}, T, g\right)>0$, then there exists a simply connected Jordan region $\Re_{*} \subset g$ and a continuous transformation $T_{*}$ defined on $\Re_{*}$ and satisfying the following conditions: (i) $\rho\left(T_{*}, T\right.$; $\left.\Re_{*}-\Re_{*}^{0}\right)=0$; (ii) $\rho\left(T_{*}, T\right.$; $\left.\Re_{*}\right)<2 \tau$; (iii) $N\left(z_{0}, T, g-\Re_{*}^{0}\right)=0$; (iv) $N\left(z_{0}, T_{*}, \Re_{*}\right)$ $=N\left(z_{0}, T_{*}, \mathfrak{N}_{*}^{\mathbf{0}}\right)=1$.

Proof. Our assumptions imply that there exists a simply connected Jordan region $\Re_{*} \subset g$ which contains the set $T^{-1}\left(z_{0}\right) \cdot g$ in its interior. Map $\Re_{*}$ onto the closed unit disk $\bar{\Re}_{*}$ with center at the origin in the $r \bar{w}$-plane by a biunique and continuous transformation given by $\bar{w}=f(w), w \in \Re_{*}$ (cf. Kerékjártó [1]). 
Define

$$
\bar{t}_{*}(\bar{w})=\left\{\begin{array}{l}
t\left(f^{-1}(\bar{w})\right) \text { if } w \in \bar{\Re}_{*}-\bar{\Re}_{*}^{0}, \\
z_{0} \text { if } \bar{w}=0, \\
\left(\bar{t}_{*}\left(\bar{w}_{*}\right)-z_{0}\right)|\bar{w}|+z_{0} \text { if } \bar{w} \in \bar{\Re}_{*}^{0}-0,
\end{array}\right.
$$

where $\bar{w}_{*}$ is the unique point of $\bar{\Re}_{*}-\bar{\Re}_{*}^{0}$ on the ray from 0 through $\bar{w}$. Finally, set $T_{*}: z=t_{*}(w)=\bar{t}_{*}(f(w))$, we $\Re_{*}$. Clearly the Jordan region $\Re_{*}$ and the transformation $T_{*}$ thus defined have the required properties.

Lemma 2. Assume that $z_{0}$ not $\in \bar{S}\left(\infty, T, \Re^{0}\right)$. If $g$ be any component of $G=G\left(\tau, z_{0}, T, \Re\right)$ for which there exists a point $w_{0} \in \bar{g}-g$ such that $\left|t\left(w_{0}\right)-z_{0}\right|<\tau$ and $N\left(z_{0}, T, g\right)>0$, then there exists a simply connected Jordan region $\Re_{*} \subset \bar{g}$ and a continuous transformation $T_{*}$ defined on $\Re_{*}$ and satisfying the following conditions: (i) $\rho\left(T_{*}, T\right.$; $\left.\left(\Re_{*}-\Re_{*}^{0}\right) \cdot \Re^{0}\right)=0$; (ii) $\rho\left(T_{*}, T\right.$; $\left.\Re_{*}\right)<2 \tau$; (iii). $N\left(z_{0}, T, g-\Re_{*}^{0}\right)=0$; (iv) $N\left(z_{0}, T_{*}, \Re_{*} \cdot \Re^{0}\right)=0$.

Using the fact that $w_{0} \in \Re-\Re^{0}$ (cf. $\$ 2.12$ ), the reader may construct the proof by methods similar to those used in the proof of Lemma 1.

2.14. Lemma. $\psi\left(z, T, \Re^{0}\right) \geqq \kappa(z, T, \Re)(c f . \S \S 1.12,2.9)$.

Proof. Clearly the lemma is established if we show that, if for a point $z_{0}$ we have $\kappa\left(z_{0}, T, \Re\right) \geqq k$, then there exists an indicator system $\left(z_{0}, T\right)$ of order $k$ in $\Re^{0}$. Since $\kappa$ is a lower semi-continuous function of $T$ (cf. $\$ 2.5$ ), there exists an $\epsilon_{0}>0$ such that, for every continuous transformation $T_{*}$ defined on $\Re$ and satisfying $\rho\left(T_{*}, T ; \Re\right)<\epsilon_{0}$, it is true that $\kappa\left(z_{0}, T_{*}, \Re\right) \geqq k$. Set $\tau=\epsilon_{0} / 3$ and $\epsilon=\epsilon_{0} / 6=\tau / 2$. Now there exists a continuous transformation $T_{\epsilon}$ defined on $\Re$ and satisfying $\rho\left(T_{\epsilon}, T, \Re\right)<\epsilon$ and $z_{0}$ not $\in \widetilde{\mathfrak{S}}\left(\infty, T_{\epsilon}, \Re\right)\left({ }^{33}\right)$. Clearly just a finite number of the components of the set $G\left(\tau, z_{0}, T_{\epsilon}, \Re\right)$ (cf. §2.12) contain points of $T_{\epsilon}^{-1}\left(z_{0}\right)$-denote by $g_{1}, \cdots, g_{l}$ those for which $\left|t_{\epsilon}(w)-z_{0}\right|=\tau$ for $w \in \bar{g}_{i}-g_{i}$ and for $i=1, \cdots, l$; denote by $g_{l+1}, \cdots, g_{m}$ those for each of which there exists a point $w_{i} \in \bar{g}_{i}-g_{i}$ such that $\left|t_{\epsilon}\left(w_{i}\right)-z_{0}\right|<\tau$ for $i=l+1, \cdots, m$. Evidently each of the $g_{i}$ for $i=1, \cdots, l$ satisfies the assumptions of Lemma 1 in $\$ 2.13$, and each of $g_{i}$ for $i=l+1, \cdots, m$ satisfies the assumptions of Lemma 2 in $\$ 2.13$. Thus there exist $m$ simply connected Jordan regions $\Re_{i}$ and $m$ continuous transformations $T_{i}: z=t_{i}(w)$ defined on $\Re_{i}$ and satisfying the conditions set forth in these lemmas. Since the $\bar{g}_{i}$ are nonoverlapping, so are the $\Re_{i}$ for $i=1, \cdots, m$. Set $\Omega=\Re-\sum_{i=1}^{m} \Re_{i}$. Define

$$
T_{\#:} \quad z=t \#(w)= \begin{cases}t_{\epsilon}(w) & \text { if } w \in \Omega ; \\ t_{i}(w) & \text { if } w \in \Re_{i}, \quad i=1, \cdots, m .\end{cases}
$$

Evidently $T_{f}$ is a continuous transformation defined on $\Re$ and satisfying the

${ }^{(33)}$ See $\left({ }^{30}\right)$. 
following conditions: (i) $\rho\left(T_{\sharp}, T ; \Omega\right)<\epsilon$; (ii) $\rho\left(T_{\#}, T\right.$ : $\left.\Re\right)<\epsilon_{0}$; (iii) $N\left(z_{0}, T_{\sharp}, \Re^{0}\right)$ $=l$. In view of (ii), (iii) and $\$ 2.10$, it follows that $k \leqq \kappa\left(z_{0}, T_{\#}, \Re\right.$ ) $=N\left(z_{0}, T_{\sharp}, \mathcal{N}\left(T_{\#}, \Re^{0}\right)\right) \leqq l$. Let $\delta$ be a positive number such that $O\left(\delta, t_{\sharp}, \Re\right)<\epsilon$ (cf. §2.3). Obviously one may choose Jordan regions $\Re_{\# i}$ (not necessarily simply connected) such that $\Re_{i} \subset \Re_{\# i} \subset g_{i}$ and $\Re_{\# i}-\Re_{\# i}^{0}$ lies in a $\delta$-neighborhood of $\bar{g}_{i}-g_{i}$ for $i=1, \cdots, l$. Since each of the $\Re_{\# i}$ clearly contains exactly one point $w_{\# i} \in T_{\#}^{-1}\left(z_{0}\right)$ in its interior and none on its boundary (cf. $\$ 2.13$ ), it follows that $\mu\left(z_{0}, T_{\#}, \Re_{\sharp i}\right)=j\left(w_{\# i}, T_{\sharp}\right)$ for $i=1, \cdots, l$ (cf. 2.10$)$. On the other hand, it is clear from the choice of the $\Re_{\sharp i}$ and from (i) above that $\epsilon\left(z_{0}, T_{\sharp}, \Re_{\sharp i}\right)>\epsilon>\rho\left(T, T_{\sharp} ; \Re_{\sharp i}-\Re_{\sharp i}^{0}\right)$ for $i=1, \cdots, l$ (cf. $\left.\$ 2.7\right)$. Thus, by Lemma 2 in $\$ 2.7$ it follows that $\mu\left(z_{0}, T, \Re_{\sharp i}\right)=\mu\left(z_{0}, T_{f}, \Re_{\sharp i}\right)$ for $i=1, \cdots, l$. Since clearly $T_{\sharp}^{-1}\left(z_{0}\right) \cdot \Re^{0}=\sum_{i=1}^{l} w_{\sharp i}$, and $\kappa\left(z_{0}, T_{\sharp}, \Re\right) \geqq k$, it follows that at least $k$ of the points $w_{\# i}$ are in the set $\mathcal{N}\left(T_{*}, \Re^{0}\right)$. Thus, for some $k$ of the Jordan regions $\Re_{\sharp i}$, it is true that $\mu\left(z_{0}, T, \Re_{\sharp i}\right) \neq 0$; these $k$ mutually exclusive Jordan regions constitute an indicator system $\left(z_{0}, T\right)$ of order $k$ in $\Re^{0}$. So the lemma is proven.

2.15. Lemma. If $\Re_{*}$ be any indicator region $\left(z_{0}, T\right)$ in $\Re^{0}$, then there exists an indicator region $\Re_{\sharp} \subset R_{*}^{0}$ such that $M\left(t(w)-z_{0}, \Re_{\#}\right) \leqq \frac{1}{2} M\left(t(w)-z_{0}, \Re_{*}\right)$ (cf. $\$ 2.3)$.

Proof. Set $\tau=\frac{1}{2} \epsilon\left(z_{0}, T, \Re_{*}\right.$ ) (cf. $\left.\$ 2.7\right)$; clearly $\tau>0$. Consider the set $G_{*}=G\left(\tau, z_{0}, T, \Re \cdot \Re_{*}\right)(\mathrm{cf} . \S 2.12)$. Since it is clear that $G_{*} \in \mathcal{S}_{*}^{0}$, it follows that but a finite number of the components of $G_{*}$ contain points of $T^{-1}\left(z_{0}\right)$-denote these components by $g_{1}, \cdots, g_{k}$. Since $\left|t(w)-z_{0}\right|=\tau>0$ for $w \in \bar{g}_{i}-g_{i}$ and for $i=1, \cdots, k$ (cf. $\$ 2.12$ ), it follows that one may choose in each $g_{i}$ a Jordan region $\Re_{i}$ containing the set $T^{-1}\left(z_{0}\right) \cdot g_{i}$ in its interior. By Lemma 5 in $\S 2.7$ it follows that $\mu\left(z_{0}, T, \Re^{*}\right)=\sum_{i=1}^{k} \mu\left(z_{0}, T, \Re_{i}\right)$. Now since $\mu\left(z_{0}, T, \Re_{*}\right) \neq 0$ (cf. $\$ 1.15)$, it follows that for some $\Re_{i}$-denote it by $\Re_{\sharp}-\mu\left(z_{0}, T, \Re_{\sharp}\right) \neq 0$. Clearly $\Re_{\#}$ is an indicator region $\left(z_{0}, T\right)$ with the required property.

2.16. Lemma. Every indicator region $(z, T)$ in $\Re^{0}$ contains an essential maximal model continuum of $z$ under $T$ in its interior; hence $\phi\left(z, T, \Re^{0}\right) \geqq \psi\left(z, T, \Re^{0}\right)$ (cf. §2.9).

Proof. If $\Re_{0}$ be any indicator region $\left(z_{0}, T\right)$, the lemma in $\$ 2.15$ insures the existence of a sequence $\Re_{1}, \cdots, \Re_{n}, \cdots$ of indicator regions $\left(z_{0}, T\right)$ such that $\Re_{n+1} \subset \Re_{n}^{0}$ for $n=0,1,2, \cdots$, and $\lim M\left(t(w)-z_{0}, \Re_{n}\right)=0$. The reader will verify without trouble that the set $\prod_{n=0}^{\infty} \Re_{n}$ is an essential maximal model continuum for $z_{0}$ under $T$ in $\Re_{0}^{0}$.

2.17. In view of the results in $\$ \S 2.9,2.14,2.16$ the theorem stated in $\$ 2.6$ is now proven. Moreover, from the definitions of $\kappa(z, T, \Re)$ (cf. $\$ 1.12$ ) and of $\kappa\left(z, T, \Re^{0}\right)$ (cf. $\$ 1.13$ ), we have, using the theorem in $\$ 2.6$, the following

Compatibility theorem. $\kappa(z, T, \Re)=\kappa\left(z, T, \Re^{0}\right)$. 
2.18. In the sequel we shall assume that $T$ is a bounded continuous transformation defined on a bounded domain $\mathcal{D}$ in the $w$-plane (cf. $\$ 1.13$ ). Then $\kappa(z, T, \mathcal{D})$ (cf. $\S 1.13$ ) possesses the following strong lower semi-continuity properties.

TheOREM. Given a sequence of domains $\mathcal{D}_{n}$ which will fill up $\mathcal{D}$ from the interior $\left({ }^{34}\right)$, and a sequence of continuous transformations $T_{n}$ defined on $\mathcal{D}_{n}$ and such that, for every closed set $F$ in $D$ it is true that $\lim \rho\left(T_{n}, T ; F\right)=0$. Then, for every $z, \lim \inf \kappa\left(z, T_{n}, \mathcal{D}_{n}\right) \geqq \kappa(z, T, \mathcal{D})$.

Proof. Clearly it is sufficient to show that, if $\kappa(z, T, \mathcal{D}) \geqq k$, then $\kappa\left(z, T_{n}, \mathcal{D}_{n}\right) \geqq k$ for all $n$ sufficiently large. If $\kappa(z, T, \mathcal{D}) \geqq k$, it follows by the theorem in $\$ 1.17$ and by the definition of essential maximal model continua for $z$ under $T$ (cf. $\$ 1.16)$, that there exists in $\mathcal{D}$ an indicator system $\subseteq$ of order $k$ for $z$ under $T$. Since the $\mathcal{D}_{n}$ fill up $\mathcal{D}$ from the interior, and since the Jordan regions $\Re \in \subseteq$ are closed, it follows that $\subseteq$ is an indicator system $(z, T)$. of order $k$ in $\mathcal{D}_{n}$ for all $n$ sufficiently large. But, from the lemma in $\$ 2.8$ and the assumption that $\lim \rho\left(T_{n}, T ; \Re\right)=0$ for every $\Re \in \mathfrak{S}$, it follows that $\mathfrak{S}$ is also an indicator system $\left(z, T_{n}\right)$ of order $k$ in $\mathcal{D}_{n}$ for all $n$ sufficiently large. Consequently, by the lemma in $\$ 2.16$ and the theorem in $\$ 1.17$ it follows that $\kappa\left(z, T_{n}, \mathcal{D}_{n}\right) \geqq k$ for all $n$ sufficiently large, and the theorem is established.

2.19. We turn, next, to the task of verifying the results stated in $\$ 1.19$; indeed, we shall establish more comprehensive results than those which have been stated there. Our results depend upon the

Lemma. If $\Re_{1}$ and $\Re_{2}$ be any two indicator regions $(z, T)$ in $\mathcal{D}$ (cf. $\left.\$ 1.15\right)$ which contain the same essential maximal model continuum $\sigma(z, T)(c f . \S 1.16)$, and are contained in a neighborhood of $\sigma(z, t)$ which is free of points belonging to other essential maximal model continua of $z$ under $T\left({ }^{35}\right)$, then $\mu\left(z, T, \Re_{1}\right)$ $=\mu\left(z, T, \Re_{2}\right)$.

Proof. First, assume that $\Re_{2} \subset \Re_{1}^{0}$. Consider the closed set $\Re_{1}-\Re_{2}^{0}$; clearly this set consists of a finite number of Jordan regions $\Re_{3}, \cdots, \Re_{k}$, such that: $\boldsymbol{\epsilon}\left(z, T, \Re_{i}\right)>0$ for $i=3, \cdots, k$, and no $\Re_{i}$ for $i=3, \cdots, k$ contains any essential maximal model continuum for $z$ under $T$; thus (cf. $\$ 2.16$ ), it follows that $\mu\left(z, T, \Re_{i}\right)=0$ for $i=3, \cdots, k$. But by Lemma 5 in $\$ 2.7$, it is true that $\mu\left(z, T, \Re_{1}\right)=\sum_{i=2}^{k} \mu\left(z, T, \Re_{i}\right)=\mu\left(z, T, \Re_{2}\right)$. Thus the lemma is established in this special case. In the general case, since $\sigma(z, T)$ is an essential maximal model continuum in the interior of $\Re_{1} \cdot \Re_{2}$, there exists (cf. $\$ 1.16$ ) in the interior of $\Re_{1} \cdot \Re_{2}$ an indicator region $\Re_{3}$ containing, of course, $\sigma(z, T)$. The preceding reasoning shows that $\mu\left(z, T, \Re_{1}\right)=\mu\left(z, T, \Re_{3}\right)=\mu\left(z, T, \Re_{2}\right)$, and the lemma is now established.

${ }^{(34)}$ See ${ }^{(22)}$.

(35) If $\sigma(z, T)$ consists of a single point $w$, then clearly $w \in \mathcal{N}(T, \mathcal{D})$. 
2.20. The assertions made in $\$ 1.19$ now follow as a corollary to the lemma in $\$ 2.19$. Moreover, we may now introduce a general local index as follows: If $\sigma(z, T)$ be any essential maximal model continuum which has a neighborhood free of points belonging to other essential maximal model continua of $z$ under $T$, then the quantity $\mu(z, T, \Re)$ is independent of the choice of an indicator region $\Re$ for $z$ under $T$ in this neighborhood $\left({ }^{36}\right)$ by the preceding lemma; we denote its value by $j_{*}(\sigma, T)$. Clearly, if $w$ be any point of $\mathcal{N}(T, \mathcal{D})$ (cf. $\S 1.18$ - - that is, if $w$ constitutes an essential maximal model continuum $\sigma$ then $j_{*}(\sigma, T)=j(w, T)$ (cf. $\left.\$ 1.19\right)$. Thus the general local index coincides with the essential local index on the set $\mathcal{N}(T, \mathcal{D})$. It is quite interesting to observe that, while the general local index is topologically and aesthetically more beautiful, it is the essential local index which plays the fundamental rôle in our applications (cf. Chapter IV). This happens, of course, because of metric assumptions (cf. Chapter IV) which reduce the essential maximal model continua which do not consist of single points to insignificance. But one interesting and enlightening fact concerning the general local index is contained in the

THEOREM. If $\Re$ be any Jordan region in $\mathcal{D}$ containing but a finite number of essential maximal model continua $\sigma_{1}(z, T), \cdots, \sigma_{k}(z, T)$ and for which $\epsilon(z, T, \Re)>0$ (cf. §2.7), then $\mu(z, T, \Re)=\sum_{i=1}^{\boldsymbol{k}} j_{*}\left(\sigma_{i}, T\right)$.

Proof. In $\Re^{0}$ choose $k$ mutually exclusive indicator regions $\Re_{i}$ for $z$ under $T$ such that $\sigma_{i} \subset \Re_{i}$ for $i=1, \cdots, k$. The closed set $\Re-\sum_{i=1}^{k} \Re_{i}^{0}$ consists of a finite number of Jordan regions $\Re_{k+1}, \cdots, \Re_{l}$, such that $\epsilon\left(z, T, \Re_{i}\right)>0$ for $i=k+1, \cdots, l$. In view of the lemmas in $\$ 2.16$ and $\$ 2.19$ it is evident that

$$
\mu\left(z, T, \Re_{i}\right)=\left\{\begin{array}{l}
j_{*}\left(\sigma_{i}, T\right) \text { for } i=1, \cdots, k, \\
0 \text { for } i=k+1, \cdots, l .
\end{array}\right.
$$

But by Lemma 5 in $\S 2.7$ it follows that $\mu(z, T, \Re)=\sum_{i=1}^{l} \mu\left(z, T, \Re_{i}\right)$. Thus $\mu(z, T, \Re)=\sum_{i=1}^{k} j_{*}\left(\sigma_{i}, T\right)$, and the theorem is proven.

2.21. The following corollary to the results in $\$ 2.20$ will be useful in the applications of our theory (cf. Chapter IV).

TheOREM. Given a bounded continuous transformation $T$ defined on a Jordan region $\Re(c f . \S 1.8)$. Let $\bar{E}$ be any set in the z-plane satisfying the conditions: (i) $|\bar{E} \cdot \bar{\Omega}(\infty, T, \Re)|=0$; (ii) $\left|\bar{E} \cdot T\left(\Re-\Re^{0}\right)\right|=0$; (iii) $\kappa(z, T, \Re)=N\left(z, T, \mathcal{E} \cdot \Re^{0}\right)$ a.e. on $\bar{E}$. Then $\mu(z, T, \Re)=\nu\left(z, T, \Re^{0}\right)$ a.e. on $\bar{E}(c f . \S \S 1.10-1.14,1.31)$.

Proof. In view of the assumptions, for almost every point $z \in \bar{E}$ it is true that $\kappa(z, T, \Re)=N\left(z, T, \mathcal{E} \cdot \Re^{0}\right)<+\infty$, and that $\epsilon(z, T, \Re)>0$ (cf. $\S 2.7$ ). For such points, we have $\nu\left(z, T, \Re^{0}\right)=\sum j(w, T)$ for $w \in \mathcal{E} \cdot \Re^{0} \cdot T^{-1}(z)$ by definition.

${ }^{(36)}$ In particular, if $\sigma(z, T)$ consists of a single point $w \in \mathcal{N}(T, \mathcal{D})$ (see $\$ 1.18$ ), then we may always choose $\Re$ as a simply connected Jordan region. However, simple examples show that generally $\Re$ may not be chosen as a simply connected Jordan region. 
On the other hand, every maximal model continuum which is essential for such a point $z$ (cf. $\$ 1.16$ ) consists of a single point which is clearly in $\mathcal{N}$ (cf. $\S 1.18)$. In view of the facts stated in $\$ 2.20$, the theorem is now obvious.

2.22. For the applications (cf. Chapter IV) we shall need to know that the sets $\mathcal{E}(T, \mathcal{D})$ and $\mathcal{N}(T, \mathcal{D})$ (cf. $\S 1.18$ ) are Borel sets, and that the function $j(w, T)(\mathrm{cf} . \$ 1.19)$ is a Baire function. We proceed to develop point-set identities which will reveal these facts (cf. \$\$2.23-2.26).

2.23 . Let $n$ be any positive integer, $m$ any integer. Denote by $E_{m, n}$ the set of all points $w \in \mathcal{D}$ for each of which there exists a Jordan region $\Re \subset \mathcal{D}$ satisfying the following conditions: (i) $w \in \Re^{0}$; (ii) $\Re$ is contained in the open disk with center $w$ and radius $1 / n$; (iii) $\mu(t(w), T, \Re)=m$. From Lemma 2 in $\$ 2.7$ it follows that $E_{m, n}$ is an open set. Clearly $E_{m, n} \supset E_{m, n+1}$ for $n=1,2, \cdots$. The reader will easily verify that

$$
\varepsilon(T, \mathcal{D})=\prod_{n=1}^{\infty} \sum_{m \neq 0} E_{m, n}
$$

2.24. For purposes in the sequel, we need another tool which we now introduce. Given a bounded domain $\mathcal{D}$ in the $w$-plane, choose an oriented $\left({ }^{37}\right)$ square $Q$ containing $\mathcal{D}$ in its interior. Let $D_{p_{j}}$ denote a subdivision of $Q$ into $p_{j}^{2}$ congruent (oriented) squares $s$, where $p_{j}$ is the $j$ th positive prime. If, now, $w$ be any point in $\mathscr{D}$, it follows that there exists a $j_{0}=j_{0}(w)$ such that, for every $j>j_{0}$ the point $w$ is comprised in the interior of some square of $D_{p_{j}}$ (see Radó $[5$, p. 1.97]).

2.25. Given a bounded continuous transformation $T$ defined on a bounded domain $\mathcal{D}$ (cf. $\$ 1.13$ ), let $U$ denote the set of all points $w \in \mathcal{D}$ each of which has a neighborhood $\mathfrak{R}(w)$ such that $\mathfrak{R}(w)-w$ contains no essential maximal model continuum for $t(w)$ under $T$ (cf. $\$ 1.16)$. The reader will easily verify that (cf. $\S 1.11,2.24)$

$$
U=\sum_{j=1}^{\infty} \sum_{8 \in D_{p_{j}, s^{0}} \subset \mathcal{D}}\left[T^{-1}(\bar{\Re}(0, T, s))-T^{-1}(\bar{\Re}(z, T, s))\right] \cdot s^{0} .
$$

Thus $U$ is a Borel set, since the sets $\bar{\Omega}(k, T, s)$ for $k<+\infty$ are open by Lemma 1 in $\$ 2.2$, and the inverse (cf. $\$ 1.8$ ) of an open set under the continuous transformation $T$ is open.

2.26. The reader will now see without difficulty that (cf. $\$ \S 2.23,2.25$ )

$$
\mathcal{N}(T, \mathcal{D})=U \cdot \varepsilon=U \cdot \prod_{n=1}^{\infty} \sum_{m \neq 0} E_{m, n} .
$$

Further, if $\mathcal{N}_{m}=\mathcal{N}_{m}(T, \mathcal{D}), m \neq 0$, denotes the set of points $w \in \mathcal{D}$ where $j(w, T)=m($ cf. $\$ 1.19)$, then the reader will easily show that

$\left.{ }^{(37}\right)$ See ${ }^{(4)}$. 


$$
\mathcal{N}_{m}(T, \mathcal{D})=U \cdot \prod_{n=1}^{\infty} E_{m, n},
$$

$m \neq 0$.

In view of the point-set identities in $\$ \$ 2.23,2.25$ and 2.26 , the assertions made in $\$ 2.22$ are true.

2.27. Given a set $\bar{E}$ in the $z$-plane, we shall denote by $g(z, \bar{E})$ its characteristic function-that is, the function whose value is 1 for $z \in \bar{E}$ and 0 otherwise. Obviously $g(z, \bar{E})$ is a measurable function of $z$ if and only if $\bar{E}$ is a measurable set.

2.28. The reader will verify without difficulty that, if $E$ be any set in the w-plane, then (cf. $\$ \$ 1.10,2.24$ )

$$
\sum_{\boldsymbol{s} \in D_{p_{j}, s^{0}} \subset \mathcal{D}} g\left(z, T\left(s^{0} \cdot E\right)\right) \underset{j \rightarrow \infty}{\longrightarrow} N(z, T, E \cdot \mathcal{D}) .
$$

Consequently $N(z, T, E \cdot \mathcal{D})$ is a measurable function of $z$ whenever the set $T\left(s^{0} \cdot E\right)$ is measurable for every choice of the square $s$ such that $s^{0} \subset \mathcal{D}$ (cf. $\$ 2.27)$.

2.29. If $E$ be any Borel set in the w-plane, then $s^{0} \cdot E$ is a Borel set for every choice of the square $s$ in the w-plane. Consequently the set $T\left(s^{0} \cdot E\right)$ is measurable for every square $s$ (see Kuratowski $\left[1\right.$, p. 249] $\left({ }^{38}\right)$. Since the sets $\mathcal{E}(T, \mathcal{D}), \mathcal{N}(T, \mathcal{D}), \mathcal{N}_{m}(T, \mathcal{D}), m \neq 0$, are all Borel sets (cf. $\S \S 2.22-2.26$ ), we have the

THEOREM. If $B$ be any Borel set in the w-plane, then the functions $N(z, T, \mathcal{E} \cdot B), N(z, T, \mathcal{N} \cdot B), N\left(z, T, \mathcal{N}_{m} \cdot B\right), m \neq 0$, are all measurable.

2.30. For the applications (cf. Chapter IV), we want to discuss the measurability of $\nu(z, T, \mathcal{D})$ (cf. $\S 1.31)$. We have the

Lemma. $\nu(z, T, \mathcal{D})=N\left(z, T, \mathcal{N}_{+1} \cdot \mathcal{D}\right)-N\left(z, T, \mathcal{N}_{-1} \cdot \mathcal{D}\right)$ for $z$ not $\in \overline{\mathfrak{S}}(\infty, T, \mathcal{E} \cdot \mathcal{D})+T\left(\mathcal{N}_{*}\right)$.

Proof. Our assumptions imply that $\nu(z, T, \mathcal{D})=\sum j(w, T)$ for $w \in \mathcal{E} \cdot \mathcal{D} \cdot T^{-1}(z)$, and that $|j(w, T)| \leqq 1$ for $w \in \mathcal{E} \cdot \mathcal{D} \cdot T^{-1}(z)$ (cf. $\left.\S \S 1.16,1.20\right)$. Thus the identity is obvious.

Since $N(z, T, \mathcal{E} \cdot \mathcal{D})$ is a measurable function of $z$ (cf. $\$ 2.29)$, it follows that the set $\overline{\mathfrak{S}}(\infty, T, \mathcal{E} \cdot \mathcal{D})$ is measurable. Now we have $\nu(z, T, \mathcal{D})=0$ for $z \in \bar{S}(\infty, T, \mathcal{E} \cdot \mathcal{D})$. In $\$ 2.39$ we shall show that the set $T\left(\mathcal{N}_{*}\right)$ is denumerable. Consequently we have the

THEOREM. $\nu(z, T, \mathcal{D})$ is a measurable function of $z$.

2.31. We have yet to establish the fact that the set $\mathcal{N}_{*}$ defined in $\$ 1.20$

(38) Since, in later chapters, we consider only transformations which satisfy certain metric restrictions, we could very well do without this general theorem on the measurability of the continuous image of a Borel set. 
is denumerable. In order to do this, we require some results concerning singlevalued continuous $k$ th roots of a complex function of a complex variable. If $t(w)$ is a single-valued continuous function of $w$ on a connected set $\mathfrak{E}$, then we say that a function $f(w)$ is a single-valued continuous $k$ th root of $t(w)$ on $\subseteq$ if (i) $f(w)$ is single-valued and continuous on $\mathfrak{E}$, and (ii) $f(w)^{k}=t(w)$ on $\mathfrak{E}$.

2.32. Lemma. Let $\sqrt{5}$ denote a connected set in the w-plane. If $t(w)$ be any bounded continuous function defined on $(\mathfrak{S}$ for which there exists a single-valued continuous kth root $f(w)$ defined on $\mathfrak{夭}$, then $O(f, \mathbb{E}) \leqq 3[O(t, \sqrt{ })]^{1 / k}(c f . \S 2.3)\left({ }^{39}\right)$.

Proof. For $k=1$ the lemma is obvious, since then $f(w)=t(w), w \in \mathcal{E}$ (cf. $\S 2.31)$; consequently $O(f, \mathfrak{E})=O(t$, (E). So assume that $k>1$. It is now convenient to consider two cases. I. First, assume that

$$
M(t, \mathfrak{E})>2 O(t, \mathfrak{E}) \text {. }
$$

Consider the continuous transformation $T$ defined by $z=t(w), w \in \mathbb{S}$ (cf. $\$ 1.8$ ). It is clear that $T(\mathfrak{S})$ is contained in a closed disk $\mathfrak{D}$ of radius $O(t, \mathfrak{E})$ whose center is at a distance exceeding $2 O(t$, $\mathfrak{E})$ from the origin in the $z$-plane. Hence $z=0$ is exterior to $\mathfrak{D}$ and we have a single-valued continuous $k$ th root of $z$ in $\mathfrak{D}$ which we denote by $g(z), z \in \mathfrak{D}$. Clearly $g(t(w)), w \in \mathfrak{C}$, is a singlevalued continuous $k$ th root of $t(w)$ on $\mathbb{E}$. Thus $g(t(w))=a f(w), w \in \mathfrak{C}$, where $a$ is a $k$ th root of unity; hence it is clear that $O(f, \mathfrak{E})=O(g, T(\mathfrak{E})) \leqq O(g, \mathfrak{D})$. Now $g(z)$ is an analytic function of $z$ in $\mathfrak{D}$ and therefore we have, for any two points $z^{\prime}, z^{\prime \prime}$ in $\mathfrak{D}$,

$$
g\left(z^{\prime \prime}\right)-g\left(z^{\prime}\right)=\int_{z^{\prime}}^{z^{\prime \prime}} g^{\prime}(z) d z,
$$

where the path of integration may be chosen as a straight segment joining $z^{\prime}$ and $z^{\prime \prime}$. Evidently

$$
g^{\prime}(z)=\frac{1}{k[g(z)]^{k-1}} .
$$

In view of (1), (2), (3) and the fact that $k>1$, we have

$$
O(f, \mathfrak{C}) \leqq O(g, \mathfrak{D}) \leqq M\left(g^{\prime}, \mathfrak{D}\right) 2 O(t, \mathfrak{C}) \leqq \frac{2}{k}[O(t, \mathfrak{C})]^{1 / k} \leqq[O(t, \mathfrak{C})]^{1 / k} .
$$

Thus the lemma is established in this case. II. Next, assume that $M(t, \mathfrak{E})$ $\leqq 2 O(t$, (5) . Then clearly (cf. $\$ 2.3$ )

$$
O(f, \mathfrak{C}) \leqq 2 M(f, \mathfrak{C}) \leqq 2[M(t, \mathfrak{C})]^{1 / k} \leqq 2^{1+1 / k}[O(t, \mathfrak{C})]^{1 / k} \leqq 3[O(t, \mathfrak{E})]^{1 / k},
$$

since $k>1$. The lemma is now established.

(39) Simple examples show that the integer 3 may not be replaced by a smaller integer. 
2.33. Lемма. Let $\Re$ denote any bounded convex region $\left.{ }^{40}\right)$ in the w-plane. If $t(w)$ be a continuous function defined on $\Re$ for which there exists a single-valued continuous kth root $f(w)$ defined on $\Re$, then $O(\delta, f, \Re) \leqq 3[O(\delta, t, \Re)]^{1 / k}$ for every positive number $\delta(c f . \S 2.3)\left({ }^{41}\right)$.

Proof. Given $\delta>0$, let $w^{\prime}$ and $w^{\prime \prime}$ be any two points of $\Re$ satisfying $\left|w^{\prime}-w^{\prime \prime}\right| \leqq \delta$. Denote by $C$ the closed line segment with end points $w^{\prime}$ and $w^{\prime \prime}$. Now $C \subset \Re$ since $\Re$ is convex. Thus it follows by the lemma in $\$ 2.32$ that

$$
\left|f\left(w^{\prime}\right)-f\left(w^{\prime \prime}\right)\right| \leqq O(f, C) \leqq 3[O(t, C)]^{1 / k} \leqq 3[O(\delta, t, \Re)]^{1 / k} .
$$

Consequently $O(\delta, f, \Re) \leqq 3[O(\delta, t, \Re)]^{1 / k}$, as asserted.

2.34. Lemma. Let $\Re$ denote any Jordan region in the w-plane. If $T$ be a continuous transformation defined on $\Re$ for which $\mu(0, T, \Re)= \pm k, k>0$, and the set $T^{-1}(0)$ consists of a single point $w_{0} \in \Re^{0}$, then $t(w)$ possesses a single-valued continuous kth root $f(w)$ in $\Re$.

Proof. Let $l$ denote a closed line segment joining $w_{0}$ to the boundary of $\Re$. In the simply connected domain $\Re^{0}-l$ it is true that $t(w) \neq 0$; consequently $\left({ }^{42}\right)$ there exists a single-valued continuous argument $\phi(w)$ for $t(w)$ in $\Re^{0}-l$. Now

$$
t(w)=|t(w)|[\cos \phi(w)+i \sin \phi(w)], \quad w \in \Re^{0}-l .
$$

Set

$$
g(w)=|t(w)| 1 / k\left[\cos \frac{\phi(w)}{k}+i \sin \frac{\phi(w)}{k}\right], \quad w \in \Re^{0}-l .
$$

Using Lemma 1 in $\$ 2.7$ and the assumption that $\mu(0, T, \Re)= \pm k$, the reader will easily show that, for every $w_{*} \in \Re, \lim g(w)$ for $w \in \Re^{0}-l, w \rightarrow w_{*}$ exists. Define

$$
f\left(w_{*}\right)=\lim g(w), \quad \text { for } w \in \Re^{0}-l, w \rightarrow w_{*} \in \Re .
$$

The reader will find that $f(w)$ is a single-valued continuous $k$ th root of $t(w)$ on $\Re$.

2.35. Lemma. Let $\Re$ be any bounded convex region in the w-plane. Let there be given continuous transformations

$$
T: \quad z=t(w), \quad T_{n}: \quad z=t_{n}(w), \quad w \in \Re,
$$

which satisfy the following conditions: (i) $\lim \rho\left(T_{n}, T ; \Re\right)=0$; (ii) $T_{n}^{-1}(0)$ con-

${ }^{(40)}$ A bounded convex region is always simply connected and bounded by a Jordan curve.

(41) Indeed, since every simply connected Jordan region is topologically equivalent to a bounded convex region, this result is also true for any simply connected Jordan region. Consequently the results in $\$ \S 2.35,2.36,2.37$ are true for any simply connected Jordan region, but we do not need this fact.

${ }^{(42}$ ) We use here the so-called monodromy theorem (see Kerékjártó [1, p. 175]). 
sists of a single point $w_{n} \in \Re^{0}$; (iii) $\mu\left(0, T_{n}, \Re\right)= \pm k, k>0$. Then $t(w)$ possesses a single-valued continuous kth root $f(w)$ on $\Re$.

Proof. In view of the assumptions on the $T_{n}$ it follows by the lemma in $\$ 2.34$ that each $t_{n}(w)$ possesses a single-valued continuous $k$ th $\operatorname{root} f_{n}(w)$ on $\Re$. Since $\lim \rho\left(T_{n}, T ; \Re\right)=0$, it follows that the functions $t_{n}(w)$ are uniformly bounded $\left({ }^{43}\right)$ and equi-continuous $\left({ }^{44}\right)$ on $\Re$. Using the lemma in $\$ 2.33$ one shows that the functions $f_{n}(w)$ are also uniformly bounded and equi-continuous on $\Re$. It follows then, by the theorem of Arzelà, that there exists a subsequence of the $f_{n}(w)$ which converges uniformly to a (continuous) function $f(w)$ on $\Re$. Since $f_{n}(w)^{k}=t_{n}(w)$ for every $n$, it follows that $f(w)^{k}=t(w), w \in \Re$. That is, $f(w)$ is a single-valued continuous $k$ th root of $t(w)$ on $\Re$.

2.36. Lemma. Let $T$ be a continuous transformation defined on a bounded convex region $\Re$ in the w-plane. Assume that $z=0$ is a point of the $z$-plane having exactly one essential maximal model continuum under $T$ in $\Re$, and this consists of a single point $w_{0} \in \Re^{0}$ for which $j\left(w_{0}, T\right)= \pm k, k>0$ (cf. \$1.19). Then there exists $a \tau>0$ such that $\kappa(z, T, \Re) \geqq k$ for every $z$ satisfying $0<|z|<\tau$.

Proof. Since $\kappa(0, T, \Re)=1$ (cf. §2.6), it follows from the definition of $\kappa$ (cf. \$1.12) that there exists a sequence of continuous transformations $T_{n}$ defined on $\Re$ and satisfying the following conditions: (i) $\lim \rho\left(T_{n}, T ; \Re\right)=0$; (ii) $T_{n}^{-1}(0)$ consists of a single point $w_{n} \in \Re^{0}$; (iii) $\mu\left(0, T_{n}, \Re\right)= \pm k$ (cf. Lemma $2, \S 2.7)$. But then, from the lemma in $\$ 2.35$ it follows that $t(w)$ possesses a single-valued continuous $k$ th root $f(w)$ on $\Re$. Consider the continuous transformation $T_{*}$ defined by $z=f(w), w \in \Re$. The reader will verify without difficulty that the only essential maximal model continuum of $z=0$ under $T_{*}$ consists of the single point $w_{0}$ and that $\mu\left(0, T_{*}, \Re\right)= \pm 1$. Thus we have $\kappa\left(0, T_{*}, \Re\right)=1$ (cf. §2.6), and consequently (cf. §2.5) there exists a $\tau_{*}>0$ such that $\kappa\left(z, T_{*}, \Re\right) \geqq 1$ for every $z$ satisfying $|z|<\tau_{*}$. Set $\tau=\tau_{*}^{\kappa}$. We assert that $\kappa(z, T, \Re) \geqq k$ for every $z$ satisfying $0<|z|<\tau$. For let $z_{0}$ be any point such that $0<\left|z_{0}\right|<\tau$; then $z_{0}$ has exactly $k$ distinct $k$ th roots $z_{1}, \cdots, z_{k}$. Clearly $\left|z_{i}\right|<\tau_{*}$ and consequently we have $\kappa\left(z_{i}, T_{*}, \Re\right) \geqq 1$, for $i=1, \cdots, k$. Thus (cf. §2.6) each $z_{i}$ possesses at least one essential maximal model continuum $\sigma_{i}=\sigma\left(z_{i}, T_{*}\right)$ in $\Re^{0}$. Since the $z_{i}$ are distinct, so are the $\sigma_{i}$ for $i=1, \cdots, k$. Now clearly $\sigma_{i} \subset T^{-1}\left(z_{0}\right)$ for $i=1, \cdots, k$. Consider the functions

$$
g_{i}(w)=f(w)^{k-1}+f(w)^{k-2} z_{i}+\cdots+z_{i}^{k-1}, \quad w \in \Re,
$$

for $i=1, \cdots, k$. Evidently each $g_{i}(w)$ is continuous on $\Re$, and $g_{i}(w)=k z_{i}^{k-1} \neq 0$ for $w \in \sigma_{i}$ and for $i=1, \cdots, k$. Consequently there exists a neighborhood $\mathfrak{N}_{i}$

$\left.{ }^{43}\right)$ The sequence of functions $f_{n}(w)$ is said to be uniformly bounded on $\Re$ if there exists a finite constant $M$ such that $M\left(f_{n}, \Re\right)<M$ for every $n$.

${ }^{(44)}$ The sequence of functions $f_{n}(w)$ is said to be equi-continuous on $\Re$ if, for every $\epsilon>0$, there exists a $\delta=\delta(\epsilon)>0$ such that $O\left(\delta, f_{n}, \Re\right)<\epsilon$ for every $n$. 
of $\sigma_{i}$ in $\Re$ such that $g_{i}(w) \neq 0$ for $w \in \Re_{i}$. Let $\Re_{i}$ be any indicator region $\left(z_{i}, T_{*}\right)$ (cf. $\S 1.15$ ) such that $\sigma_{i} \subset \Re_{i} \subset \Re_{i}$ for $i=1, \cdots, k$. We have the identity

$$
t(w)-z_{0}=\left(f(w)-z_{i}\right) g_{i}(w), \quad w \in \Re .
$$

Thus, by Lemma 1 and Lemma 3 in $\$ 2.7$ it follows that $\mu\left(z_{0}, T, \Re_{i}\right)$ $=\mu\left(z_{i}, T_{*}, \Re_{i}\right) \neq 0$ for $i=1, \cdots, k$. Thus each $\sigma_{i}$ is also an essential maximal model continuum $\left(z_{0}, T\right)$ in $\Re^{0}$ - that is, $\kappa\left(z_{0}, T, \Re\right) \geqq k$, as asserted.

2.37. Leмma. Let $T$ be a continuous transformation defined on a bounded convex region $\Re$ in the w-plane. Assume that $z_{0}$ is a point of the z-plane having exactly one essential maximal model continuum under $T$ in $\Re$, and this consists of a single point $w_{0} \in \Re^{0}$ for which $j\left(w_{0}, T\right)= \pm k, k>0$. Then there exists $a \tau>0$ such that $\kappa(z, T, \Re) \geqq k$ for every $z$ satisfying $0<\left|z-z_{0}\right|<\tau$.

Proof. Consider the transformation $T_{0}$ defined by the relation $z=t_{0}(w)$ $=t(w)-z_{0}, w \in \Re$, and apply the lemma in $\$ 2.36$.

2.38. Now let $T$ be a bounded continuous transformation defined on a bounded domain $\mathcal{D}$ in the w-plane (cf. $\S 1.8$ ). Given a positive integer $k$, define, for every square $s$ such that $s^{0} \subset \mathcal{D}$, the set $\bar{V}(k, s)$ to be the set of points $z$ having the following properties: (i) $z$ possesses exactly one essential maximal model continuum $\sigma(z, T)$ in $s$; (ii) $\sigma(z, T)$ consists of a single point $w \in s^{0}$; (iii) $j(w, T)= \pm k$ (cf. $\$ 1.19$ ). It follows immediately from the lemma in $\$ 2.37$ that $\bar{V}(k, s)$, for $k>1$, is an isolated set, and hence denumerable. Next, let $\bar{V}(k, \mathcal{D})$ denote the set of all points $z$ for each of which there exists a point $w \in \mathcal{N} \cdot T^{-1}(z)$ (cf. $\left.\$ 1.18\right)$ for which the essential index $j(w, T)= \pm k$. The reader will easily verify the identity (cf. \$2.24)

$$
\bar{V}(k, \mathcal{D})=\sum_{j=1}^{\infty} \sum_{s \in D_{p_{j}, s^{0}} \in \mathcal{D}} \bar{V}(k, s) .
$$

Thus, if $k>1$, it follows that the set $\bar{V}(k, \mathcal{D})$ is denumerable. Consequently the set

is also denumerable.

$$
\bar{V}=\sum_{k=2}^{\infty} \bar{V}(k, \mathcal{D})
$$

2.39. The reader will find it easy to verify the

LEMMA. $T\left(\mathcal{N}_{*}\right)=\bar{V}(c f . \$ 1.20)$.

Thus the image of $\mathcal{N}_{*}$ under $T$ is a denumerable set.

2.40. Now, for every point $z$, the set $\mathcal{N}_{*} \cdot T^{-1}(z)$ is clearly an isolated set. But obviously we have

$$
\mathcal{N}_{*}=\sum_{z \in \dot{V}} \mathcal{N}_{*} \cdot T^{-1}(z),
$$


since $\mathcal{N}_{*} \subset T^{-1}(\bar{V})$ by the lemma in $\$ 2.39$. Since $\bar{V}$ contains at most a denumerable number of points, so does $\mathcal{N}_{*}$; thus the important fact stated in $\$ 1.20$ is established.

\section{Chapter III. Metric Foundations}

3.1. We state first a few facts concerning functions of rectangles $\left({ }^{45}\right)$, due essentially $\left({ }^{46}\right)$ to Banach (see Banach [1], Radó [5]). Let $F(R)$ be a function of rectangles defined for all closed oriented rectangles in a given bounded domain $\mathcal{D}$. Then $F(R)$ is said to be of bounded variation in $\mathcal{D}$ if there exists a finite constant $M$ such that

$$
\sum_{j=1}^{m}\left|F\left(s_{j}\right)\right| \leqq M
$$

for every system of closed squares in $\mathcal{D}$ without common interior points. $F(R)$ is said to be absolutely continuous in $\mathcal{D}$ if, for every $\epsilon>0$ there exists an $\eta(\epsilon)>0$ such that

$$
\sum_{j=1}^{m}\left|F\left(s_{j}\right)\right| \leqq \epsilon
$$

for every system of closed squares in $\mathcal{D}$, without common interior points, which satisfy the condition

$$
\sum_{j=1}^{m}\left|s_{j}\right| \leqq \eta(\epsilon)
$$

If $F(R)$ is absolutely continuous and bounded on $\mathcal{D}$, then it is also of bounded variation in $\mathcal{D}$ (see Radó [5, p. 193]).

3.2. Take a point $w_{0}$ in $\mathcal{D}$ and consider all possible sequences $\left\{s_{j}\right\}$ of closed squares such that

$$
w_{0} \in s_{j} \subset \mathcal{D}
$$

and $\left|s_{j}\right| \rightarrow 0$. The least upper bound of $\lim \sup F\left(s_{j}\right) /\left|s_{j}\right|$, for all such sequences $\left\{s_{j}\right\}$, is the upper derivative $\bar{F}^{\prime}\left(w_{0}\right)$. The lower derivative is defined in a similar way. These derivatives may be equal to $\pm \infty$. They are always measurable. If the upper and lower derivatives are finite and equal at a point $w_{0}$, then their common value is the derivative $F^{\prime}\left(w_{0}\right)$ of $F(R)$ at the point $w_{0}$.

3.3. Let $S$ be a closed square comprised in $\mathcal{D}$, and let $s_{1}, \cdots, s_{m}$ be a sys-

(45) Let us state again (see $\left.{ }^{4}\right)$ ) that we consider only rectangles having sides parallel to the respective axes.

(46) The facts listed in $\$ \$ 3.1-3.5$ are not stated in exactly the same form in which the reader finds them in Banach [1], but in a form convenient for our purposes. However, the necessary modifications in the proofs of Banach are quite obvious (see Rad6 [5, p. 192] for further remarks on this subject). 
tem of closed squares without common interior points contained in $S$. We

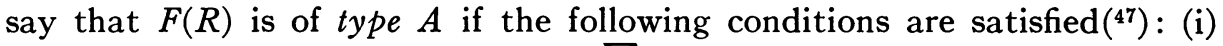
$F(s) \geqq 0$ for every square $s \subset \mathcal{D}$; (ii) $\sum_{j=1}^{m} F\left(s_{j}\right) \leqq F(S)$ for every system of squares $S, s_{1}, \cdots, s_{m}$ as described above.

If $F(R)$ is of type $A$, then its derivative $F^{\prime}(w)$ exists a.e. in $\mathcal{D}$ and is summable in every rectangle $R \subset \mathcal{D}$. Furthermore, we have, for every rectangle $R \subset \mathcal{D}$,

$$
\iint_{R} F^{\prime}(w) \leqq F(R)
$$

3.4. Given a function of rectangles $F(R)$ in $\mathcal{D}$, suppose that $F(R)$ is absolutely continuous in $\mathcal{D}$ and that the derivative $F^{\prime}(w)$ exists a.e. in $\mathcal{D}$. Then we have, for every open set $O \subset \mathcal{D}$, the relation (cf. \$2.24)

$$
\sum_{s \in D p_{j},,^{0} \subset O} F(s) \underset{j \rightarrow \infty}{\longrightarrow} \iint_{O} F^{\prime}(w) .
$$

3.5. Let us now assume only that $F(R) \geqq 0$, and that the derivative $F^{\prime}(w)$ exists a.e. in $\mathcal{D}$. Take any open set $O \subset \mathcal{D}$ and assume that

$$
l=\liminf _{l \rightarrow \infty} \sum_{s \in D_{p_{j}, s^{0} \subset O}} F(s)<+\infty .
$$

Then $F^{\prime}(w)$ is summable in $O$, and

$$
\iint_{0} F^{\prime}(w) \leqq l
$$

To prove this assertion, define the functions $\phi_{j}(w)$ as follows: if $s$ is a square such that $s^{0} \subset O, s \in D_{p_{j}}$, then $\phi_{j}(w)=F(s) /|s|$ on the interior of $s$. Otherwise $\phi_{j}(w)=0$. Then clearly

$$
\phi_{j}(w) \rightarrow F^{\prime}(w) \text { a.e. in } O
$$

and (cf. §2.24)

$$
\iint_{0} \phi_{j}(w)=\sum_{s \in D_{p_{j}, 8^{0} \subset O}} F(s) .
$$

In view of the lemma of Fatou( $\left.{ }^{48}\right),(4),(5),(6)$ imply our assertion.

3.6. Let us consider now a transformation (cf. \$1.13)

$$
T: \quad z=t(w),
$$

${ }^{(47)}$ This type of rectangle functions is not considered explicitly by Banach, but his methods (see Banach [1]) yield easily the desired results.

( $\left.{ }^{48}\right)$ The lemma of Fatou may be stated as follows. Let $\Phi_{n} \geqq 0, \Phi \geqq 0$ be measurable functions on a bounded measurable set $S$. Suppose that (i) $\Phi_{n}$ is summable on $S$; (ii) $\Phi_{n} \rightarrow \Phi$ a.e. on $S$; (iii) $\lim \inf \int_{S} \Phi_{n}<+\infty$. Then $\Phi$ is also summable on $S$, and $\int_{S} \Phi \leqq \lim \inf \int_{S} \Phi_{n}$. See, for instance, Saks $[1$, p. 29]. 
Since $t(w)$ is bounded by assumption, the set $T(\mathcal{D})$ is contained in some finite open circular disc $\bar{\Delta}$ in the $z$-plane. Let there be given, in the w-plane, a base set $B$ with the measurability properties described in $\$ 1.21$.

3.7. If $E$ is a set comprised in $\mathcal{D}$, we shall use the symbol( $\left.{ }^{49}\right) \bar{A}(E, k)$ to denote the set of those points $z$ for which $N(z, E)=k$. We have then the identity (cf. \$2.24)

(7) $\mathcal{D}-\mathscr{B} \subset T^{-1}(\bar{A}(\mathcal{D} \cdot \mathcal{B},+\infty))+\sum_{j=1}^{\infty} \sum_{8 \in D_{p_{j}, 8^{0}} \subset \mathcal{D}} s^{0} \cdot T^{-1}\left(\bar{A}\left(s^{0} \cdot \mathcal{B}, 0\right)\right)$.

If $E_{1}, \cdots, E_{m}$ are nonoverlapping sets contained in a set $E \subset \mathcal{D}$, then we have the following relations (cf. $\$ \S 1.10,2.27)$ :

$$
\begin{gathered}
\sum_{j=1}^{m} N\left(z, E_{j} \cdot \mathcal{B}\right) \leqq N(z, E \cdot \mathcal{B}), \\
\sum_{j=1}^{m} g\left(z, T\left(E_{j} \cdot \mathcal{B}\right)\right) \leqq N(z, E \cdot \mathcal{B}), \\
\sum_{j=1}^{m}\left[N\left(z, E_{j} \cdot \mathcal{B}\right)-g\left(z, T\left(E_{j} \cdot \mathcal{B}\right)\right)\right] \leqq N(z, E \cdot \mathcal{B})-g(z, T(E \cdot \mathcal{B})) .
\end{gathered}
$$

If $O$ is any open set comprised in $\mathcal{D}$, then

$$
\sum_{s \in D_{p_{j}, 8^{0} \subset O}} g\left(z, T\left(s^{0} \cdot \mathcal{B}\right)\right) \underset{j \rightarrow \infty}{\longrightarrow} N(z, O \cdot \mathcal{B}),
$$

and, a fortiori,

$$
\sum_{{ }_{80 D} D_{p_{j}, 8^{0} \subset O}} N\left(z, s^{0} \cdot \mathbb{B}\right) \underset{j \rightarrow \infty}{\longrightarrow} N(z, O \cdot \mathbb{B}) .
$$

The verification of these simple facts is left to the reader.

3.8. Since $g\left(z, T\left(s^{0} \cdot \mathbb{B}\right)\right)$ is, by assumption, measurable for every $s^{0} \subset \mathcal{D}$, it follows from (11) that $N(z, O \cdot \mathrm{B})$ is measurable for every open set $O \subset \mathcal{D}$. As a consequence, the sets $\bar{A}(O \cdot \mathcal{B}, k)(\mathrm{cf} . \S 3.7)$ are measurable.

3.9. Take now any closed set $C \subset \mathcal{D}$, and put $O=\mathcal{D}-C$. We have then $N(z, \mathcal{D} \cdot \mathbb{B})=N(z, C \cdot \mathbb{B})+N(z, O \cdot \mathbb{B})$. In view of $\S 3.8$, it follows that $N(z, C \cdot \mathbb{B})$ is measurable on the complement of the $\operatorname{set} \bar{A}(\mathcal{D} \cdot \mathcal{B},+\infty)$. Hence, if this latter set is of measure zero, then it follows that $N(z, C \cdot \mathrm{B})$ is measurable for every closed set $C \subset \mathcal{D}$.

3.10. Now let $E$ be any measurable set comprised in $\mathcal{D}$. We can write $E=e+\Gamma$, where $e \cdot \Gamma=0,|e|=0$, and $\Gamma=\sum_{j=1}^{\infty} C_{j}$, where $C_{1} \subset C_{2} \subset \cdots \subset C_{j}$ $\subset \cdots$ and every $C_{j}$ is a closed set. We have then

(49) Since the transformation $T$ is fixed throughout this chapter, we shall delete the argument $T$ from the notations introduced in Chapter I; for example, we write $N(z, E)$ instead of $N(z, T, E)$. 


$$
N(z, E \cdot \mathbb{B})=N(z, e \cdot \mathcal{B})+N(z, \Gamma \cdot \mathcal{B}), N\left(z, C_{j} \cdot \mathbb{B}\right) \underset{j \rightarrow \infty}{\longrightarrow} N(z, \Gamma \cdot \mathcal{B})
$$

In view of $\$ 3.9$, we obtain the

LEMмA. If $|T(e \cdot \mathcal{B})|=0$ whenever $|e|=0$, and if $|\bar{A}(\mathcal{D} \cdot \mathcal{B},+\infty)|=0$, then, for every measurable set $E \subset \mathcal{D}$ the function $N(z, E \cdot \mathbb{B})$ is measurable.

3.11. Let us assume now that $T$ is B.V. $B$ in $\mathscr{D}$ (cf. $\$ 1.23$ ). We can write (cf. $\$ \$ 1.10,2.24,2.27$ )

$$
\psi_{j}(z)=\sum_{z \in D_{p_{j}, z^{0}} \subset \mathcal{D}} g\left(z, T\left(s^{0} \cdot \mathbb{B}\right)\right)\left\{\begin{array}{l}
\leqq N(z, \mathcal{D} \cdot \mathcal{B}), \\
\underset{j \rightarrow \infty}{\longrightarrow} N(z, \mathcal{D} \cdot \mathcal{B}) .
\end{array}\right.
$$

Then (cf. \$1.22)

$$
\iint \psi_{j}(z)=\sum_{s \in D_{p_{j}, s^{0} \subset \mathcal{D}}} G(s) .
$$

Since $T$ is B.V. B in $\mathcal{D}$, the summation on the right side of this equality is less than a fixed finite constant independent of $j$. Hence, by the lemma of Fatou $\left({ }^{50}\right), N(z, \mathcal{D} \cdot \mathbb{B})$ is summable.

Conversely, suppose that $N(z, \mathcal{D} \cdot \mathbb{B})$ is summable. Then, for any system $s_{1}, \cdots, s_{m}$ of closed squares without common interior points and such that $s_{1}^{0}, \cdots, s_{m}^{0}$ are contained in $\mathcal{D}$, by $(9) \sum_{j=1}^{m} g\left(z, T\left(s_{j}^{0} \cdot \mathcal{B}\right)\right) \leqq N(z, \mathcal{D} \cdot \mathcal{B})$, and hence, by integration

$$
\sum_{j=1}^{m} G\left(s_{j}\right) \leqq \iint N(z, \mathcal{D} \cdot \mathbb{B})
$$

Thus $T$ is B.V. $\mathcal{B}$ in $\mathcal{D}$.

3.12. If $T$ is B.V. $\mathbb{B}$ in $\mathcal{D}$, then by $\$ 3.11$ the function $N(z, \mathcal{D} \cdot \mathcal{B})$ is summable, and hence the set $\bar{A}(\mathcal{D} \cdot \mathbb{B}, \infty)$ is of measure zero (cf. $\$ 3.7)$.

3.13. If $T$ is B.V. $\mathbb{B}$ in $\mathcal{D}$, then $N(z, \mathcal{D} \cdot \mathbb{B}$ ) is summable (cf. $\$ 3.11$ ), and hence, a fortiori, $N\left(z, R^{0} \cdot \mathbb{B}\right)$ is summable for every closed rectangle $R$ such that $R^{0} \subset \mathcal{C}$. We can consider therefore the rectangle function

$$
G^{*}(R)=\iint N\left(z, R^{0} \cdot \mathbb{B}\right)
$$

It follows then by integration from (8) that $G^{*}(R)$ is of type $A$ (cf. $\S 3.3$ ). Hence the derivative of $G^{*}(R)$ exists a.e. in $\mathcal{D}$. We shall denote this derivative by $D^{*}(w)$.

It follows by integration from (10) that the rectangle function $G^{*}(R)$ $-G(R)$ is also of type $A$, and hence that its derivative exists a.e. in $\mathcal{D}$. Con-

${ }^{(50)}$ See ${ }^{(48)}$. 
sequently the rectangle function $G(R)=G^{*}(R)-\left(G^{*}(R)-G(R)\right)$ also has a derivative a.e. in $\mathcal{D}$. We shall denote the derivative of $G(R)$ by $D(w)$.

3.14. Suppose $T$ is B.V. B in $\mathcal{D}$. Take any open set $O \subset \mathcal{D}$. Since $N(z, \mathcal{D} \cdot \mathbb{B})$ is now summable (cf. $\S 3.11$ ), it follows from (8), (9), (11), (12) that

$$
\sum_{s \in D_{p_{j}, z^{0} \subset O}} G(s) \underset{j \rightarrow \infty}{\longrightarrow} \iint N(z, O \cdot \mathcal{B}), \quad \sum_{s \in p_{p_{j}, z^{0} \subset O}} G^{*}(s) \underset{j \rightarrow \infty}{\longrightarrow} \iint N(z, O \cdot \mathcal{B}),
$$

and hence also

$$
\sum_{s \in D_{p_{j}, s^{0} \subset O}}\left(G^{*}(s)-G(s)\right) \underset{j \rightarrow \infty}{\longrightarrow} 0 .
$$

By 3.5 it follows that $D(w)$ and $D^{*}(w)$ are summable on $O$ and

$$
\iint_{o} D(w) \leqq \iint N(z, O \cdot \mathbb{B})
$$

In particular, it follows, for $O=\mathcal{D}$, that $D(w)$ is summable on $\mathscr{D}$. If we apply the result in $\$ 3.5$ to the rectangle function $G^{*}(R)-G(R)$, for $O=\mathcal{D}$, then we obtain the inequality

$$
\iint_{\mathcal{D}}\left(D^{*}(w)-D(w)\right) \leqq 0 .
$$

Since obviously $D^{*}(w)-D(w) \geqq 0$, it follows that $D^{*}(w)=D(w)$ a.e. in $\mathcal{D}$.

3.15. Lemma. Suppose T is B.V. B in D. Let $\bar{E}$ be a measurable set in the z-plane, such that $N(z, \mathcal{D} \cdot \mathbb{B})=0$ a.e. on $\bar{E}$. Then $D(w)=0$ a.e. on the set $T^{-1}(\bar{E})$.

Proof. Since $T(\mathcal{D})$ is a bounded set contained in a finite open disc $\bar{\Delta}$ (cf. $\S 3.6)$, clearly $T^{-1}(\bar{E})=T^{-1}(\bar{\Delta} \cdot \bar{E})$. Hence we can assume that $\bar{E}$ itself is bounded. Take then a sequence of open sets $\bar{O}_{n}$ such that $\bar{O}_{1} \supset \bar{O}_{2} \supset \cdots \supset \bar{O}_{n}$ $\supset \cdots \supset \bar{E}, \quad\left|\bar{O}_{n}\right| \rightarrow|\bar{E}|$. Put $T^{-1}\left(\bar{O}_{n}\right)=O_{n}, \Gamma=\prod O_{n}, T^{-1}(\bar{E})=E$. Then clearly $O_{1} \supset O_{2} \supset \cdots \supset O_{n} \supset \cdots \supset \Gamma \supset E$, and hence it is sufficient to show that $D(w)=0$ a.e. on $\Gamma$. Using $\$ 3.14$ we have

Thus

$$
\begin{aligned}
\iint_{\Gamma} D(w) & \leqq \iint_{o_{n}} D(w) \leqq \iint N\left(z, O_{n} \cdot \mathcal{B}\right)=\iint_{\bar{o}_{n}} N\left(z, O_{n} \cdot \mathcal{B}\right) \\
& \leqq \iint_{\bar{o}_{n}} N(z, \mathcal{D} \cdot \mathcal{B}) \rightarrow \iint_{\bar{E}} N(z, \mathcal{D} \cdot \mathcal{B})=0
\end{aligned}
$$

$$
\iint_{\Gamma} D(w)=0
$$

Hence $D(w)=0$ a.e. on $\Gamma$, and the lemma is proved.

3.16. The preceding result implies various corollaries. 
Corollary a. If $T$ is B.V. B in $\mathcal{D}$, and if $\bar{E}$ is a set of measure zero in the $z$-plane, then $D(w)=0$ a.e. on $T^{-1}(\bar{E})$.

Corollary b. If $T$ is B.V. B in $\mathcal{D}$, then $D(w)=0$ a.e. on $T^{-1}(\bar{A}(\mathcal{D} \cdot \mathcal{B}),+\infty)$ ) $(c f . \S \$ 3.12,3.7)$.

Corollary c. If $T$ is B.V. $\mathbb{B}$ in $\mathcal{D}$, then $D(w)=0$ a.e. on $T^{-1}(\bar{A}(\mathcal{D} \cdot \mathbb{B}), 0)$ ) (cf. §3.7).

3.17. Finally, let us observe that if $T$ is B.V. $B$ in $\mathcal{D}$ then it is clearly B.V. $B$ in every subdomain of $\mathcal{D}$. Corollary b, Corollary c, and the identity (7) imply therefore the following

Theorem. If $T$ is $B . V$. B in $\mathcal{D}$, then $D(w)=0$ a.e. on $\mathcal{D}-\mathcal{B}$.

3.18. Suppose $T$ is B.V. $B$ in $\mathcal{D}$. Consider, in the $z$-plane, a finite-valued measurable function $H(z)$. We cannot then assert that $H(t(w))$ is measurable in $\mathcal{D}$, but we shall see presently that $H(t(w)) D(w)$ is measurable in $\mathcal{D}$.

Proof. Since $T(\mathcal{D})$ is contained in a finite open disc $\bar{\Delta}$ (cf. §3.6), we can restrict ourselves to consider $H(z)$ in $\bar{\Delta}$. Since $H(z)$ is measurable there, we have a sequence of continuous functions $H_{n}(z)$ in $\bar{\Delta}$ such that $H_{n}(z) \rightarrow H(z)$ on $\bar{\Delta}-\bar{E}$, where $\bar{E}$ is some set of measure zero. Put $E=T^{-1}(\bar{E})$. The function $H_{n}(t(w))$ is continuous in $\mathcal{D}, D(w)$ is measurable in $\mathcal{D}$, and hence our assertion will be proved if we can show that

$$
H_{n}(t(w)) D(w) \rightarrow H(t(w)) D(w) \text { a.e. in } \mathcal{D} .
$$

Now (13) holds a.e. on $\mathcal{D}-E$, since there $H_{n}(t(w)) \rightarrow H(t(w))$ and $D(w)$ exists a.e. in $\mathcal{D}$. On $E$, we have $D(w)=0$ a.e. by Corollary a in $\$ 3.16$, and hence (13) holds there also.

3.19. Assume again that $T$ is B.V. $B$ in $\mathcal{D}$. Suppose $H_{1}(z), H_{2}(z)$ are finitevalued measurable functions in the z-plane, such that $H_{1}(z)=H_{2}(z)$ except on a set $\bar{E}$ of measure zero. Put $E=T^{-1}(\bar{E})$. Then the equation

$$
H_{1}(t(w)) D(w)=H_{2}(t(w)) D(w)
$$

holds a.e. on $\mathcal{D}-E$, since there $H_{1}(t(w))=H_{2}(t(w))$ and $D(w)$ exists a.e. in $\mathcal{D}$. On $E$, we have $D(w)=0$ a.e. by Corollary a in $\$ 3.16$ and hence (14) holds a.e. on $E$. Thus the equation (14) holds a.e. in $\mathcal{D}$.

3.20. Suppose now that $T$ is A.C. $B$ in $\mathcal{D}$ (cf. $\$ 1.23$ ). By $\S 3.1$ it follows that $T$ is also B.V. $B$ in $\mathcal{D}$. From $\S \S 3.13,3.14$ and $\S 3.4$ it follows then that

$$
\iint_{o} D(w)=\iint N(z, O \cdot \mathbb{B})
$$

for every open set $O \subset \mathcal{D}$, in particular, for $O=\mathcal{D}$.

3.21. Now suppose that $T$ is B.V. $B$ in $\mathcal{D}$, and that 


$$
\iint_{\mathcal{D}} D(w)=\iint N(z, \mathcal{D} \cdot \mathbb{B})
$$

We wish to show that $T$ is A.C. $B$ in $\mathcal{D}$.

Proof. Take any rectangle $R$ such that $R^{0} \subset \mathcal{D}$. We have then a sequence $\left\{R_{j}\right\}$ of rectangles such that $R, R_{1}, \cdots, R_{j}, \cdots$ have no common interior points, and such that $\sum R_{j}^{0}+R^{0}=\mathcal{D}$, except for a set of measure zero. Using $\S 3.14$ we have then

$$
\iint_{R^{0}} D(w) \leqq \iint N\left(z, R^{0} \cdot \mathcal{B}\right), \quad \iint_{R_{j}^{0}} D(w) \leqq \iint N\left(z, R_{j}^{0} \cdot \mathcal{B}\right),
$$

and hence

$$
\begin{aligned}
\iint_{\mathcal{D}} D(w) & =\iint_{R^{0}} D(w)+\sum_{j} \iint_{R_{j}^{0}} D(w) \\
& \leqq \iint N\left(z, R^{0} \cdot \mathbb{B}\right)+\sum_{j} \iint N\left(z, R_{j}^{0} \cdot \mathbb{B}\right) \\
& \leqq \iint N(z, \mathcal{D} \cdot \mathbb{B})=\iint_{\mathcal{D}} D(w) .
\end{aligned}
$$

Hence the sign of equality holds throughout in the preceding relations. In particular (cf. \$3.13),

$$
\iint_{R^{0}} D(w)=\iint N\left(z, R^{0} \cdot \mathscr{B}\right)=G^{*}(R)
$$

This shows that $G^{*}(R)$ and hence $G(R)$ (cf. $\$ 1.22$ ) are absolutely continuous in $\mathcal{D}$ (cf. §3.1). Thus $T$ is A.C. $B$ in $\mathcal{D}$.

3.22. LemmA. If $T$ is A.C. $\mathbb{B}$ in $\mathcal{D}$, and if $E$ is a set of measure zero in $\mathcal{D}$, then $T(E \cdot B)$ is also a set of measure zero.

Proof. Take a sequence of open sets $O_{n}$ in $\mathcal{D}$ such that $O_{1} \supset O_{2} \supset \cdots \supset O_{n}$ $\supset \cdots \supset E,\left|O_{n}\right| \rightarrow 0$. Using $\$ 3.20$, we have

$$
\left|T\left(O_{n} \cdot \mathbb{B}\right)\right| \leqq \iint N\left(z, O_{n} \cdot \mathbb{B}\right)=\iint_{O_{n}} D(w) \rightarrow 0 .
$$

Hence $\left|T\left(O_{n} \cdot \mathbb{B}\right)\right| \rightarrow 0$. But $T(E \cdot \mathbb{B}) \subset T\left(O_{n} \cdot \mathbb{B}\right)$ for every $n$. Thus $T(E \cdot \mathbb{B})$ is of measure zero.

3.23. Corollary. If $T$ is A.C. $\mathcal{B}$ in $\mathcal{D}$, and if $E$ is a measurable set in $\mathcal{D}$, then $N(z, E \cdot B)$ is measurable.

This follows from $\$ 3.22$ in view of $\S \S 3.10-3.12$ and $\$ 3.20$. 
3.24. We assume now that $T$ is A.C. $B$ in $\mathcal{D}$ and we proceed to prove the transformation formula of $\$ 1.29$. The proof is obtained in several steps. Let us first assume that $H(z)$ is the characteristic function of an open set $\bar{O}$ in the $z$-plane, while the set $E$ coincides with an open set $O^{*} \subset \mathcal{D}$. If we put $O=T^{-1}(\bar{O})$, then obviously the transformation formula is equivalent to the formula

$$
\iint_{O \cdot O^{*}} D(w)=\iint N\left(z, O \cdot O^{*} \cdot \mathbb{B}\right)
$$

By $\$ 3.20$, this formula is correct, since $O \cdot O^{*}$ is an open set.

3.25. Suppose next that $E$ is an open set $O^{*} \subset \mathcal{D}$, while $H(z)$ is the characteristic function of a measurable set $\bar{Z}$ in the $z$-plane. Since $T$ is a bounded continuous transformation, we can assume that $\bar{Z}$ is a bounded set. The function $H(t(w))$ is then the characteristic function of the set $Z=T^{-1}(\bar{Z})$. Take a sequence of bounded open sets $\bar{O}_{n}$ in the $z$-plane such that $\bar{O}_{1} \supset \bar{O}_{2} \supset \cdots \supset \bar{O}_{n} \supset \cdots \supset \bar{Z},\left|\bar{O}_{n}\right| \rightarrow|\bar{Z}|$. Let $H_{n}(z)$ be the characteristic function of the set $\bar{O}_{n}$. Then we clearly have $H_{n}(z) \rightarrow H(z)$ a.e. Since $\left|H_{n}(z)\right| \leqq 1$ and since $N(z, \mathcal{D} \cdot \mathbb{B})$ is summable, it follows that

$$
\iint H_{n}(z) N\left(z, O^{*} \cdot \mathbb{B}\right) \rightarrow \iint H(z) N\left(z, O^{*} \cdot \mathbb{B}\right) .
$$

Put $O_{n}=T^{-1}\left(\bar{O}_{n}\right)$. Then $H_{n}(t(w))$ is the characteristic function of $O_{n}$. By a reasoning analogous to that used in $\$ 3.18$, we see that $H_{n}(z) \rightarrow H(z)$ a.e. implies that $H_{n}(t(w)) D(w) \rightarrow H(t(w)) D(w)$ a.e. in $\mathcal{D}$. Since $D(w)$ is summable in $\mathcal{D}$ and since $\left|H_{n}(t(w))\right| \leqq 1$, it follows that

$$
\iint_{O^{*}} H_{n}(t(w)) D(w) \rightarrow \iint_{O^{*}} H(t(w)) D(w) .
$$

By $\$ 3.24$ we have, for every $n$,

$$
\iint_{O^{*}} H_{n}(t(w)) D(w)=\iint H_{n}(z) N\left(z, O^{*} \cdot \mathbb{B}\right) .
$$

(15), (16), (17) imply the desired result.

3.26. Suppose now that $E$ is an open set $O^{*} \subset \mathcal{D}$, while $H(z)$ is a bounded measurable function which takes on only a finite number of distinct values. Then $H(z)$ can be written in the form $C_{1} H_{1}(z)+C_{2} H_{2}(z)+\cdots+C_{m} H_{m}(z)$, where $C_{1}, \cdots, C_{m}$ are constants, and $H_{1}(z), \cdots, H_{m}(z)$ are the characteristic functions of measurable sets. Thus the desired result follows directly from $\$ 3.25$.

3.27. Let $E$ be an open set $O^{*} \subset \mathcal{D}$, while $H(z)$ is a bounded measurable function, say $|H(z)| \leqq L$. Then we have a sequence $\left\{H_{n}(z)\right\}$ of functions of the type considered in $\S 3.26$, such that $\left|H_{n}(z)\right| \leqq L$ and $H_{n}(z) \rightarrow H(z)$ a.e. in 
a finite open disc $\bar{\Delta}$ (cf. §3.6) which contains $T(\mathcal{D})$. The desired result follows then from $\$ 3.26$ by well-known theorems on termwise integration.

3.28. Let $E$ be any measurable set in $\mathcal{D}$, while $H(z)$ is a bounded measurable function. In $\mathcal{D}$, take a sequence $\left\{O_{n}\right\}$ of open sets such that $O_{1} \supset O_{2} \supset \cdots \supset O_{n} \supset \cdots \supset E,\left|O_{n}\right| \rightarrow|E|$. Clearly then

$$
\iint_{o_{n}} H(t(w)) D(w) \rightarrow \iint_{E} H(t(w)) D(w) .
$$

By $\$ 3.27$ we have

$$
\iint_{o_{n}} H(t(w)) D(w)=\iint H(z) N\left(z, O_{n} \cdot \mathcal{B}\right) .
$$

Put $\Gamma=\prod O_{n}$. Then obviously

$$
N\left(z, O_{n} \cdot \mathcal{B}\right)\left\{\begin{array}{l}
\leqq N(z, \mathcal{D} \cdot \mathcal{B}), \\
\rightarrow N(z, \Gamma \cdot \mathcal{B}) \text { if } N(z, \mathcal{D} \cdot \mathscr{B})<+\infty .
\end{array}\right.
$$

Since the set where $N(z, \mathcal{D} \cdot \mathbb{B})=+\infty$ is of measure zero, and since $H(z)$ is bounded, it follows that

$$
\iint H(z) N\left(z, O_{n} \cdot \mathcal{B}\right) \rightarrow \iint H(z) N(z, \Gamma \cdot \mathcal{B}) .
$$

But $|\Gamma-E|=0$, and hence, by $\$ 3.22$, we have $N(z,(\Gamma-E) \cdot \mathbb{B})=0$ a.e. Consequently

$$
N(z, \Gamma \cdot \mathbb{B})=N(z, E \cdot \mathbb{B}) \text { a.e. }
$$

The desired result follows now from (18), (19), (20), (21).

3.29. Let $E$ be a measurable set in $\mathcal{D}$, while $H(z)$ is a finite-valued, measurable, and non-negative function. For every positive integer $n$, put

$$
H_{n}(z)=\left\{\begin{array}{cl}
H(z) & \text { if } H(z) \leqq n, \\
n & \text { if } H(z)>n .
\end{array}\right.
$$

By $\$ 3.28$ we have then

$$
\iint_{E} H_{n}(t(w)) D(w)=\iint H_{n}(z) N(z, E \cdot \mathbb{B}) .
$$

Since all the functions involved are non-negative, the desired result follows by the lemma of Fatou( $\left.{ }^{51}\right)$.

3.30. Let, finally, $E$ be a measurable set in $\mathcal{D}$, while $H(z)$ is a finite-valued, measurable function, and such that one of the two integrals

(51) See ${ }^{(48)}$. 


$$
\iint_{E} H(t(w)) D(w), \quad \iint H(z) N(z, E \cdot \mathbb{B})
$$

exists. Then one of the integrals

$$
\iint_{E}|H(t(w))| D(w), \quad \iint|H(z)| N(z, E \cdot \mathcal{B})
$$

exists also. By $\S 3.29$, both of these integrals exist then, and

$$
\iint_{E}|H(t(w))| D(w)=\iint|H(z)| N(z, E \cdot B) .
$$

The existence of the integrals (23) implies, however, the existence of both of the integrals (22). If we put $H^{*}(z)=|H(z)|-H(z)$, then $H^{*}(z) \geqq 0$, hence $\$ 3.29$ applies to $H^{*}(z)$ and we have

$$
\iint_{E} H^{*}(t(w)) D(w)=\iint H^{*}(z) N(z, E \cdot \mathbb{B}),
$$

where the existence of these integrals follows from the preceding remarks. The desired result is now obtained by subtracting (25) from (24).

\section{Chapter IV. The generalized Jacobian}

4.1. In order to establish the theorems stated in $\$ \$ 1.30-1.36$, we need a sequence of lemmas, which we now proceed to consider. The transformation (cf. §1.13)

$$
T: \quad z=t(w), \quad w \in \mathcal{D},
$$

will be fixed until further notice, and hence we shall delete the argument $T$ from the notations introduced in Chapter $I\left({ }^{52}\right)$. The essential set $\varepsilon$, defined in $\$ 1.18$, will play a fundamental part in the sequel.

4.2. Given a rectangle $R$ such that $R^{0} \subset \mathcal{D}$, we shall denote by $g_{1}(z, R)$ the characteristic function of the set of those points $z$ where $\nu\left(z, R^{0}\right) \neq 0$ (cf. $\$ 1.31$ ). We have then obviously

$$
g_{1}(z, R) \leqq N\left(z, \varepsilon \cdot R^{0}\right) .
$$

Let us take now a subdivision $\left({ }^{53}\right)$ of $R$ into a finite number of rectangles $R_{1}, \cdots, R_{m}$. The reader will easily verify that

$$
\sum_{i=1}^{m}\left[N\left(z, \varepsilon \cdot R_{i}^{0}\right)-g_{1}\left(z, R_{i}\right)\right] \leqq N\left(z, \varepsilon \cdot R^{0}\right)-g_{1}(z, R) .
$$

(52) See $\left.{ }^{49}\right)$.

(53) A subdivision of $R$ consists of a finite number of rectangles $R_{1}, R_{2}, \cdots, R_{m}$ without common interior points such that $R=R_{1}+R_{2}+\cdots+R_{m}$. 
4.3. Given a rectangle $R$ such that $R^{0} \subset \mathcal{D}$, we define $g_{2}(z, R)$ as the characteristic function of the set $T\left(\varepsilon \cdot R^{0}\right)$ - that is, of the set where $N\left(z, \varepsilon \cdot R^{0}\right) \neq 0$; we define $g_{3}(z, R)$ as the characteristic function of the set where $\kappa\left(z, R^{0}\right) \neq 0$ (cf. §1.13). We introduce then the rectangle functions $\left({ }^{54}\right)$

$$
\begin{gathered}
G_{1}(R)=\iint g_{1}(z, R), \quad G_{2}(R)=\iint g_{2}(z, R)=\left|T\left(\varepsilon \cdot R^{0}\right)\right|, \\
G_{3}(R)=\iint g_{3}(z, R) .
\end{gathered}
$$

In case $N(z, \mathcal{E} \cdot \mathcal{D})$ is summable, we define

$$
G_{2}^{*}(R)=\iint N\left(z, \varepsilon \cdot R^{0}\right)
$$

In case $\kappa(z, \mathcal{D})$ is summable, we define

$$
G_{3}^{*}(R)=\iint \kappa\left(z, R^{0}\right)
$$

The derivatives of these rectangle functions, if they exist, will be denoted by $D_{1}(w), D_{2}(w), D_{3}(w), D_{2}^{*}(w), D_{3}^{*}(w)$ respectively. We define also a generalized Jacobian

$$
\mathcal{F}(w)=j(w) D_{2}(w),
$$

where $j(w)$ is the local index defined in $\$ 1.19$. Since, except for a denumerable set, we have $j(w)=0,+1,-1$ (cf. $\$ 1.20)$, it follows that, except for a denumerable set, $|\mathcal{F}(w)| \leqq D_{2}(w)$ whenever $D_{2}(w)$ exists.

4.4. Lemma. If $T$ is B.V. $\mathcal{E}$ in $\dot{\mathcal{D}}$ (cf. §1.23), then (i) $D_{2}(w)$ and $D_{2}^{*}(w)$ exist a.e. in $\mathcal{D}$, are summable there, and $D_{2}(w)=D_{2}^{*}(w)$ a.e. in $\mathcal{D}$; (ii) $D_{1}(w)$ exists a.e. in $\mathcal{D}$ and is summable there.

Proof. The assertion (i) is a direct consequence of $\$ 3.14$, with $B=\varepsilon$. To prove (ii) we observe that integration of (27) yields

$$
\sum_{i=1}^{m}\left(G_{2}^{*}\left(R_{i}\right)-G_{1}\left(R_{i}\right)\right) \leqq G_{2}^{*}(R)-G_{1}(R) .
$$

This means that the rectangle function $G_{2}{ }^{*}(r)-G_{1}(r)$ is of type $A$ (cf. $\S 3.3$ ). Thus $G_{1}(r)$ is the difference of the functions $G_{2}^{*}(r)$ and $G_{2}^{*}(r)-G_{1}(r)$, each of which is of type $A$ (cf. $\$ 3.13)$. Hence, by $\$ 3.3$, the derivative $D_{1}(w)$ exists a.e. in $\mathcal{D}$. Integration of $(26)$ yields $G_{1}(R) \leqq G_{2}^{*}(R)$ and hence $D_{1}(w) \leqq D_{2}^{*}(w)$ a.e. in $\mathcal{D}$. Since $D_{2}^{*}(w)$ is summable in $\mathcal{D}$, the summability of $D_{1}(w)$ follows.

$\left({ }^{54}\right)$ The measurability of the functions involved in the following formulas follows from $\S \S 2.18,2.22-2.30$. 
4.5. Lemмa. If $\kappa(z, \mathcal{D})$ is summable, then the derivatives $D_{2}(w), D_{2}^{*}(w)$, $D_{3}(w), D_{3}^{*}(w)$ exist a.e. in $\mathcal{D}$, are summable in $\mathcal{D}$, and $D_{2}(w)=D_{2}^{*}(w)=D_{3}(w)$ $=D_{3}^{*}(w)$ a.e. in $\mathcal{D}$.

Proof. Since $N(z, \mathcal{E} \cdot \mathcal{D}) \leqq \kappa(z, \mathcal{D})$ (cf. $\S \S 1.13,1.18)$, it follows that $N(z, \mathcal{E} \cdot \mathcal{D})$ is summable, and hence, by $\S 3.11$, it follows that $T$ is B.V. $\mathcal{E}$ in $\mathscr{D}$. In view of $\S 4.4$ it is sufficient to prove that $D_{3}(w), D_{3}^{*}(w)$ exist and are equal to $D_{2}(w)=D_{2}^{*}(w)$ a.e. in $\mathcal{D}$. Now take any rectangle $R$ such that $R^{0} \subset \mathcal{D}$, and let $R_{1}, R_{2}, \cdots, R_{m}$ be rectangles forming a subdivision $\left({ }^{55}\right)$ of $R$. We have then

$$
\sum_{i=1}^{m} \kappa\left(z, R_{i}^{0}\right) \leqq \kappa\left(z, R^{0}\right),
$$

by $\$ 1.17$. Integration yields (cf. $\$ 4.3$ )

$$
\sum_{i=1}^{m} G_{3}^{*}\left(R_{i}\right) \leqq G_{3}^{*}(R) .
$$

Thus $G_{3}^{*}(R)$ is of type $A$ (cf. $\S 3.3$ ), and hence $D_{3}^{*}(w)$ exists a.e. in $\mathcal{D}$. Consider again a rectangle $R$ such that $R^{0} \subset \mathcal{D}$, and define (cf. \$2.24)

$$
\phi_{j}(z)=\sum_{s \in D_{p_{j}, s^{0} \subset R^{0}}} \kappa\left(z, s^{0}\right) .
$$

We have then, by $\S 1.17, \phi_{j}(z) \leqq \kappa\left(z, R^{0}\right) \leqq \kappa(z, \mathcal{D})$ and, whenever $\kappa(z, \mathcal{D})<+\infty$ and hence a.e., $\phi_{j}(z) \rightarrow N\left(z, \varepsilon \cdot R^{0}\right)$. These last two relations guarantee termwise integrability of the sequence $\phi_{j}(z)$ and we obtain

$$
\sum_{\varepsilon \in D_{p_{j}, 8^{0} \subset R^{0}}} G_{3}^{*}(s) \underset{j \rightarrow \infty}{\longrightarrow} \iint N\left(z, \varepsilon \cdot R^{0}\right)=G_{2}^{*}(R) .
$$

We have therefore, by $\$ 3.5$,

$$
\iint_{R^{0}} D_{3}^{*}(w) \leqq G_{2}^{*}(R)
$$

Since this holds for every rectangle, and thus, in particular, for every square, in $\mathcal{D}$, it follows that $D_{3}^{*}(w) \leqq D_{2}^{*}(w)$ a.e. in $\mathcal{D}$. Since clearly $G_{2}^{*}(R) \leqq G_{3}^{*}(R)$ (cf. $\S 4.3$ ), we also have $D_{2}^{*}(w) \leqq D_{3}^{*}(w)$ a.e. in $\mathcal{D}$. Hence $D_{2}^{*}(w)=D_{3}^{*}(w)$ a.e. in $\mathcal{D}$. Furthermore, since clearly $G_{2}(R) \leqq G_{3}(R) \leqq G_{3}^{*}(R)$, the upper and lower derivatives (cf. $\$ 3.2)$ of $G_{3}(R)$ are comprised a.e. between $D_{2}(w)$ and $D_{3}^{*}(w)$. Since we already know that $D_{2}(w)=D_{2}^{*}(w)=D_{3}^{*}(w)$ a.e. in $\mathcal{D}$, it follows that $D_{3}(w)$ exists a.e. in $\mathcal{D}$ and is equal to $D_{2}(w)$ a.e. in $\mathcal{D}$. Summing up, we have $D_{3}^{*}(w)=D_{2}^{*}(w)=D_{2}(w)=D_{3}(w)$ a.e. in $\mathcal{D}$, as asserted.

4.6. Lemma. If $T$ is A.C. $\mathcal{E}$ in $\mathcal{D}$ and if $\kappa(z, \mathcal{D})$ is summable, then $D_{1}(w)=D_{2}^{*}(w)$ a.e. in $\mathcal{D}$.

${ }^{(55)}$ See ${ }^{(53)}$. 
Proof. Let $R$ be a rectangle such that $R^{0} \subset \mathcal{D}$. Let us consider the auxiliary function

$$
\alpha_{j}(z)=\sum_{s \in D p_{j}, s^{0} \subset R^{0}} g_{1}(z, s)
$$

Clearly $\alpha_{j}(z) \leqq N\left(z, \mathcal{E} \cdot R^{0}\right) \leqq \kappa(z, \mathcal{D})$ and $\alpha_{j}(z) \rightarrow N\left(z, \varepsilon \cdot R^{0}\right)$, provided that $\kappa(z, \mathcal{D})<+\infty$. Since $\kappa(z, \mathcal{D})$ is summable (and hence $\kappa(z, \mathcal{D})<+\infty$ a.e.), it follows that the sequence $\alpha_{j}(z)$ can be integrated termwise. Thus we obtain

$$
\sum_{s \in D_{p_{j}: s^{0} \subset R^{0}}} G_{1}(s) \underset{j \rightarrow \infty}{\longrightarrow} G_{2}^{*}(R) .
$$

The inequality $g_{1}(z, R) \leqq N\left(z, \mathcal{E} \cdot R^{0}\right)$ yields, by integration,

$$
G_{1}(R) \leqq G_{2}^{*}(R) \text {. }
$$

Since $T$ is A.C. $\mathcal{E}$ in $\mathcal{D}$, we have, by $\S 3.20$ and $\S 3.14$,

$$
G_{2}^{*}(R)=\iint_{R} D_{2}^{*}(w)
$$

Thus the rectangle function $G_{1}(R)$ has the following properties: (i) $G_{1}(R)$ is absolutely continuous (by (30) and (31)); (ii) the derivative $D_{1}(w)$ of $G_{1}(R)$ exists a.e. in $\mathcal{D}$ (by $\$ 4.4$ ). In view of $\$ 3.4$, these properties (i) and (ii) imply that

$$
\sum_{s \in D_{p_{j^{\prime}}, 8^{0} \subset R^{0}}} G_{1}(s) \underset{j \rightarrow \infty}{\longrightarrow} \iint_{R} D_{1}(w) .
$$

(29), (31), (32) imply that

$$
\iint_{R} D_{1}(w)=\iint_{R} D_{2}^{*}(w) .
$$

Since $R$ is any rectangle in $\mathcal{D}$, this equality implies that $D_{1}(w)=D_{2}^{*}(w)$ a.e. in $\mathcal{D}$. $\$ 4.3)$.

4.7. Lemma. If $\kappa(z, \mathcal{D})$ is summable, then $|\mathcal{F}(w)|=D_{2}(w)$ a.e. in $\mathcal{D}(c f$.

Proof. Since $N(z, \mathcal{E} \cdot \mathcal{D}) \leqq \kappa(z, \mathcal{D})$, it follows first, by $\S 3.11$, that $T$ is B.V. $\mathcal{E}$ in $\mathcal{D}$, and hence $D_{2}(w)$ and $\mathcal{F}(w)=j(w) D_{2}(w)$ exist a.e. in $\mathcal{D}$ (cf. $\$ \S 3.13,4.3$ ). Let now $w_{0}$ be a point such that $D_{2}\left(w_{0}\right)$ and $\mathcal{F}\left(w_{0}\right)$ exist and $D_{2}\left(w_{0}\right) \neq\left|\mathcal{f}\left(w_{0}\right)\right|$. Then surely $D_{2}\left(w_{0}\right) \neq 0$ and $j\left(w_{0}\right) \neq \pm 1$. Except for points which belong to a set of measure zero, these conditions imply that $w_{0} \in \mathcal{E}$ (since $D_{2}(w)=0$ a.e. on $\mathcal{D}-\varepsilon$ by $\S 3.17$ ), and that $w_{0}$ is not in $\mathcal{N}$ (since $j(w)= \pm 1$ on $\mathcal{N}$ except for a denumerable set, by $\S 1.20)$. Hence $w_{0} \in \mathcal{E}-\mathcal{N}$. But $T(\mathcal{E}-\mathcal{N})$ is a subset of the set of those points $z$ where $\kappa(z, \mathcal{D})=+\infty$ (cf. $\S 1.18$ ). This latter set being 
of measure zero (since $\kappa(z, \mathcal{D})$ is summable), it follows by $\S 3.16$ that $D_{2}(w)=0$ a.e. on $\varepsilon-\mathcal{N}$. Since $D_{2}\left(w_{0}\right) \neq 0$, the lemma follows.

4.8. We assume now that $T \in K_{1}(\mathcal{D})$ (cf. §1.32). Applying the theorem of $\$ 1.29$ with $B=\mathcal{E}$, we have the fundamental formula

$$
\iint_{E} H(t(w)) D_{2}(w)=\iint H(z) N(z, \varepsilon \cdot E)
$$

(where $E$ is any measurable set in $\mathcal{D}$ and $H(z)$ is any finite-valued measurable function) provided only that the integrals involved exist. We also know that the existence of one of the integrals involved implies the existence of the other (cf. §1.29).

4.9. Assuming again that $T \in K_{1}(\mathcal{D})$, let us consider a finite-valued measurable function $H(z)$ such that $H(t(w)) \mathcal{F}(w)$ is summable in $\mathcal{D}$. Since $\mathcal{F}(w)=0$ a.e. on $\mathcal{D}-\mathcal{N}(\mathrm{cf} . \S \S 1.18,1.19,3.16,3.17)$, this means summability on the set $\mathcal{N}=\mathcal{N}_{+1}+\mathcal{N}_{-1}+\mathcal{N}_{*}$ (cf. $\S \S 1.20,2.26$ ). But $\mathcal{N}_{*}$ is denumerable (cf. $\$ 1.20$ ). Hence, using $\$ 4.8$,

$$
\begin{aligned}
\iint_{\mathcal{D}} H(t(w)) \mathcal{F}(w) & =\iint_{\mathcal{N}_{+1}} H(t(w)) \mathcal{F}(w)+\iint_{\mathcal{N}_{-1}} H(t(w)) \mathcal{F}(w) \\
& =\iint_{\mathcal{N}_{+1}} H(t(w)) D_{2}(w)-\iint_{\mathcal{N}_{-1}} H(t(w)) D_{2}(w) \\
& =\iint H(z) N\left(z, \mathcal{N}_{+1}\right)-\iint H(z) N\left(z, \mathcal{N}_{-1}\right) \\
& =\iint H(z)\left[N\left(z, \mathcal{N}_{+1}\right)-N\left(z, \mathcal{N}_{-1}\right)\right] .
\end{aligned}
$$

By $\$ 2.30, N\left(z, \mathcal{N}_{+1}\right)-N\left(z, \mathcal{N}_{-1}\right)=\nu(z, \mathcal{D})$ a.e., and the theorem of $\S 1.32$ is proved. The theorem in $\$ 1.34$ now follows directly by $\$ 2.21$.

4.10. Concerning the class $K_{2}(\mathcal{D})$, defined in $\$ 1.34$, inspection of the lemmas in $\$ \S 4.5,4.6,4.7$ yields the

Theorem. If $T \in K_{2}(\mathcal{D})$, then

$$
D_{1}(w)=D_{2}(w)=D_{3}(w)=D_{2}^{*}(w)=D_{3}^{*}(w)=|\mathcal{F}(w)|
$$

a.e. in D.

4.11. We proceed now to prove the closure theorem (cf. \$1.35) for the class $K_{2}(\mathcal{D})$. Using the assumptions and notations of $\$ 1.35$ let us take a Jordan region $\Re \subset \mathcal{D}$. By assumption ${ }^{(56}$ )

$\left.{ }^{(56}\right)$ Of course, $t_{n}(w)$ will be defined in $\Re$ only if $n$ is sufficiently large. In the course of the proof, the subscript $n$ refers to the transformation $T_{n}$. For example, $\varepsilon_{n}=\mathcal{E}\left(T_{n}, \mathcal{D}_{n}\right)$. 


$$
t_{n}(w) \rightrightarrows t(w) \text { on } \Re, \iint_{\Re}\left|\mathcal{F}(w)-\mathcal{F}_{n}(w)\right| \rightarrow 0 \text {, }
$$

and hence, a fortiori,

$$
\begin{gathered}
t_{n}(w) \rightrightarrows t(w) \text { on } \Re^{0}, \\
\iint_{\Re^{0}}\left|\mathcal{F}(w)-\mathcal{F}_{n}(w)\right| \rightarrow 0 .
\end{gathered}
$$

Thus (cf. §2.18) a.e.

(34) $N\left(z, T, \mathcal{E} \cdot \Re^{0}\right) \leqq \kappa\left(z, T, \Re^{0}\right) \leqq \lim \inf \kappa\left(z, T_{n}, \Re^{0}\right)=\lim \inf N\left(z, T_{n}, \mathcal{E}_{n} \cdot \Re^{0}\right)$.

Using (33), $\$ 4.7$ and $\$ 4.8$, we have

(35) $\iint N\left(z, T_{n}, \varepsilon_{n} \cdot \Re^{0}\right)=\iint_{\Re^{0}}\left|\mathcal{F}_{n}(w)\right| \rightarrow \iint_{\Re^{0}}|\mathcal{F}(w)| \leqq \iint_{\mathcal{D}}|\mathcal{F}(w)|$.

By the lemma of Fatou $\left({ }^{57}\right),(34)$ and (35) imply that $\kappa\left(z, T, \Re^{0}\right)$ is summable, and

$$
\iint \kappa\left(z, T, \Re^{0}\right) \leqq \iint_{\mathcal{D}}|\mathcal{F}(w)|
$$

If we apply this inequality to a sequence of Jordan regions which fill up $\mathcal{D}$ from the interior (cf. $\$ 1.13$ ), it follows, by $\$ 2.18$, and by the lemma of Fatou, that $\kappa(z, T, \mathcal{D})$ is summable, and

$$
\iint \kappa(z, T, \mathcal{D}) \leqq \iint_{\mathcal{D}}|\mathcal{F}(w)|
$$

Since $\kappa(z, T, \mathcal{D})$ is summable, it follows that $T$ is B.V. $\mathcal{E}$ in $\mathcal{D}$ and by $\S \S 3.14$, 4.3 and (36) we can write

$$
\begin{aligned}
\iint_{\mathcal{D}}|\mathcal{F}(w)| & \leqq \iint_{\mathcal{D}} D_{2}(w) \leqq \iint N(z, T, \mathcal{E} \cdot \mathcal{D}) \\
& \leqq \iint \kappa(z, T, \mathcal{D}) \leqq \iint_{\mathcal{D}}|\mathcal{F}(w)| .
\end{aligned}
$$

Hence the sign of equality holds throughout, and in view of $\S 3.21$ the theorem follows.

4.12. We shall need the following well-known theorems in Lebesgue theory. Let $\bar{E}$ be a bounded measurable set in the z-plane. Let $f_{n}(z), f(z)$ be summable functions on $\bar{E}$, such that $f_{n}(z) \rightarrow f(z)$ a.e. in $\bar{E}$. Let us say that the

(5i) See ${ }^{(48)}$. 
sequence $\left\{f_{n}(z)\right\}$ satisfies the condition $U$ on $\bar{E}$ if, for every $\epsilon>0$, there exists a $\delta=\delta(\epsilon)>0$ such that

$$
\iint_{\bar{e}^{-}}\left|f_{n}(z)\right| \leqq \epsilon
$$

for every measurable subset $\bar{e}$ of $\bar{E}$ such that $|\bar{e}|<\delta$ (see de la Vallée Poussin $[1])$. Then the following statements are true: (i) we have

$$
\iint_{\bar{S}} f_{n}(z) \rightarrow \iint_{\bar{S}} f(z)
$$

for every measurable subset $\bar{S}$ of $\bar{E}$ if and only if the sequence $\left\{f_{n}(z)\right\}$ satisfies the condition $U$ on $\bar{E}$; (ii) we have

$$
\iint_{\bar{E}}\left|f_{n}(z)\right| \rightarrow \iint_{\bar{E}}|f(z)|
$$

if and only if the sequence $\left\{f_{n}(z)\right\}$ satisfies the condition $U$ on $\bar{E}$.

4.13. As an immediate corollary to the preceding statements, it follows that

$$
\iint_{\bar{E}}\left|f_{n}(z)-f(z)\right| \rightarrow 0
$$

if and only if

$$
\iint_{\bar{E}}\left|f_{n}(z)\right| \rightarrow \iint_{\bar{E}}|f(z)|
$$

4.14. We proceed now to prove the theorem of $\$ 1.36$. Using the notations and assumptions of that theorem, we first note that $\mu\left(z, T_{n}, \Re\right) \rightarrow \mu(z, T, \Re)$ a.e. in the $z$-plane. Indeed, this holds if $z$ is not in $T\left(\Re-\Re^{0}\right)$ (cf. $\left.\$ 2.7\right)$, and we have $\left|T\left(\Re-\Re^{0}\right)\right|=0$ by assumption. We note also that all the functions $\mu\left(z, T_{n}, \Re\right), \mu(z, T, \Re)$ are equal to zero outside of a conveniently chosen circular disc (cf. §3.6). In view of $\$ 4.13$ it is therefore sufficient to prove that

$$
\iint\left|\mu\left(z, T_{n}, \Re\right)\right| \rightarrow \iint|\mu(z, T, \Re)|
$$

Let $E$ denote the set of those points $w \in \Re^{0}$ for which $t(w)$ is a point of $T\left(\Re-\Re^{0}\right)$. Since $\left|T\left(\Re-\Re^{0}\right)\right|=0$ by assumption, it follows by $\S 3.16$ that $\mathcal{F}(w)=0$ a.e. on $E$. Choosing $H(z)$ as the function $\left({ }^{58}\right) \operatorname{sgn} \mu(z, T, \Re)$, and $H_{n}(z)$ as the function $\operatorname{sgn} \mu\left(z, T_{n}, \Re\right)$, we have, for any fixed $z$ not in $T\left(\Re-\Re^{0}\right)$, the relation $H_{n}(z)=H(z)$ for large values of $n$ (cf. $\left.\$ 2.7\right)$. Thus $H_{n}(z) \rightarrow H(z)$ a.e. in the $z$-plane. But a much stronger relation holds. Indeed, let $z_{0}$ be any point

${ }^{\left({ }^{5}\right)}$ If $a$ is a real number, then $\operatorname{sgn} a=+1,-1,0$ according as $a>0, a<0, a=0$. 
not in $T\left(\Re-\Re^{0}\right)$. Let $\bar{\gamma}$ denote a small closed circular disc which " contains $z_{0}$ but contains no point of $T\left(\Re-\Re^{0}\right)$. Clearly we have an $n_{0}$ such that $H_{n}(z)=H(z)$ for $z \in \bar{\gamma}, n>n_{0}$. From this we infer that, if $z$ is not in $T\left(\Re-\Re^{x}\right)$ and if $z_{n} \rightarrow z$, then $H_{n}\left(z_{n}\right) \rightarrow H(z)$, and hence

$$
H_{n}\left(t_{n}(w)\right) \rightarrow H(t(w)) \text {, if } w \text { not } \in E .
$$

Set

$$
\begin{aligned}
I_{n}^{\prime} & =\iint_{\Re^{0}} H_{n}\left(t_{n}(w)\right)\left(\mathcal{F}_{n}(w)-\mathcal{F}(w)\right), \\
I_{n}^{\prime \prime} & =\iint_{\Re^{0-E}}\left(H_{n}\left(t_{n}(w)\right)-H(t(w))\right) \mathcal{F}(w), \\
I_{n}^{\prime \prime \prime} & =\iint_{E}\left(H_{n}\left(t_{n}(w)\right)-H(t(w))\right) \mathcal{F}(w) .
\end{aligned}
$$

Then clearly

$$
\iint_{\Re_{0}} H_{n}\left(t_{n}(w)\right) \mathcal{F}_{n}(w)=I_{n}^{\prime}+I_{n}^{\prime \prime}+I_{n}^{\prime \prime \prime}+\iint_{\Re^{0}} H(t(w)) \mathcal{F}(w) .
$$

We have, since $\left|H_{n}\right| \leqq 1$,

$$
\left|I_{n}^{\prime}\right| \leqq \iint_{\Re^{0}}\left|\mathcal{F}_{n}(w)-\mathcal{F}(w)\right|
$$

hence $I_{n}^{\prime} \rightarrow 0$ as a direct consequence of our assumptions. By (37) we have, since $\left|H_{n}-H\right| \leqq 2$ and $\mathcal{F}(w)$ is summable, $I_{n}^{\prime \prime} \rightarrow 0$. Since, by a preceding remark, $\mathcal{f}(w)=0$ a.e. on $E$, we have $I_{n}{ }^{\prime \prime \prime}=0$. Thus

$$
\iint_{\Re^{0}} H_{n}\left(t_{n}(w)\right) \mathcal{F}_{n}(w) \rightarrow \iint_{\Re^{0}} H(t(w)) \mathcal{F}(w) .
$$

By the transformation formula of $\$ 1.34$ this relation yields directly

$$
\iint H_{n}(z) \mu\left(z, T_{n}, \Re\right) \rightarrow \iint H(z) \mu(z, T, \Re) .
$$

This completes the proof, since by the definition of $H_{n}(z)$ and of $H(z)$ we have

$$
H_{n}(z) \mu\left(z, T_{n}, \Re\right)=\left|\mu\left(z, T_{n}, \Re\right)\right|, \quad H(z) \mu(z, T, \Re)=|\mu(z, T, \Re)| .
$$

Chapter V. The ordinary Jacobian

5.1. In this chapter, we shall consider a bounded continuous transformation $T$ defined on a bounded domain $\mathcal{D}$ by relations of the form (cf. $\$ 1.8$ )

$$
T: x=x(u, v), \quad y=y(u, v), \quad(u, v) \in \mathcal{D} \text {. }
$$


We assume that the partial derivatives $x_{u}, x_{v}, y_{u}, y_{v}$ exist a.e. in $\mathcal{D}$, and we denote the ordinary Jacobian by

$$
J(w)=J(u, v)=x_{u} y_{v}-x_{v} y_{u} .
$$

5.2. Let $w_{0}=u_{0}+i v_{0}$ (cf. $\S 1.8$ ) be a point of $\mathcal{D}$ and let $\left\{s_{n}\right\}$ be a sequence of closed squares comprised in $\mathcal{D}$. We shall say that the point $w_{0}$ and the sequence $\left\{s_{n}\right\}$ jointly satisfy the condition $C\left(\left\{s_{n}\right\}, w_{0}\right)$ if the following facts

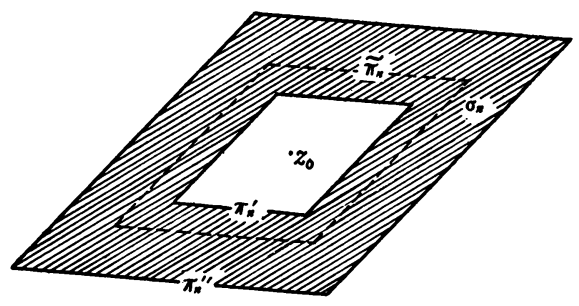

FIG. 1

hold: (i) each $s_{n}$ is an oriented square with center at $w_{0}$; (ii) $\lim \left|s_{n}\right|=0$; (iii) $J\left(w_{0}\right)$ exists; (iv) if $b_{n}$ denotes the boundary of $s_{n}$ - that is, $b_{n}=s_{n}-s_{n}^{0}$ and if we put

$$
\begin{aligned}
& \xi\left(s_{n}, w_{0}\right)=\max _{(u, v) \epsilon b_{n}} \frac{\left|x(u, v)-x\left(u_{0}, v_{0}\right)-\left(u-u_{0}\right) x_{u}\left(u_{0}, v_{0}\right)-\left(v-v_{0}\right) x_{v}\left(u_{0}, v_{0}\right)\right|}{\left[\left(u-u_{0}\right)^{2}+\left(v-v_{0}\right)^{2}\right]^{1 / 2}}, \\
& \eta\left(s_{n}, w_{0}\right)=\max _{(u, v) \in b_{n}} \frac{\left|y(u, v)-y\left(u_{0}, v_{0}\right)-\left(u-u_{0}\right) y_{u}\left(u_{0}, v_{0}\right)-\left(v-v_{0}\right) y_{v}\left(u_{0}, v_{0}\right)\right|}{\left[\left(u-u_{0}\right)^{2}+\left(v-v_{0}\right)^{2}\right]^{1 / 2}},
\end{aligned}
$$

then $\lim \xi\left(s_{n}, w_{0}\right)=0, \lim \eta\left(s_{n}, w_{0}\right)=0$.

5.3. Our assumption that $x_{u}, x_{v}, y_{u}, y_{v}$ exist a.e. in $\mathcal{D}$ (cf. §5.1) implies that, for almost every point $w \in \mathcal{D}$ there exists a sequence $\left\{s_{n}\right\}$ of squares comprised in $\mathcal{D}$ for which the condition $C\left(\left\{s_{n}\right\}, w\right)$ is satisfied (see Radó [5, p. 219]).

5.4. Consider now a point $w_{0}$ and a sequence $\left\{s_{n}\right\}$ satisfying the condition $C\left(\left\{s_{n}\right\}, w_{0}\right)$. Let us introduce the auxiliary affine transformation

$$
\tilde{T}: \begin{aligned}
& x=x\left(u_{0}, v_{0}\right)+\left(u-u_{0}\right) x_{u}\left(u_{0}, v_{0}\right)+\left(v-v_{0}\right) x_{v}\left(u_{0}, v_{0}\right), \\
& y=y\left(u_{0}, v_{0}\right)+\left(u-u_{0}\right) y_{u}\left(u_{0}, v_{0}\right)+\left(v-v_{0}\right) y_{v}\left(u_{0}, v_{0}\right), \quad(u, v) \in \mathcal{D} .
\end{aligned}
$$

By a wholly elementary reasoning $\left({ }^{59}\right)$, we obtain the following statements concerning the relation between $T$ and $\tilde{T}$.

Let us denote by $z_{0}$ the point $T\left(w_{0}\right)=\tilde{T}\left(w_{0}\right)$. I. Suppose, first, that $J\left(w_{0}\right) \neq 0$. For brevity, denote $\widetilde{T}\left(s_{n}\right)$ by $\tilde{\pi}_{n}$. Then $\tilde{\pi}_{n}$ is a parallelogram with

${ }^{\left({ }^{5}\right)}$ See, for instance Radó [1] for a full discussion of practically the same situation. 
center at $z_{0}$. Since $\tilde{T}$ is an affine transformation, we have

$$
\left|\tilde{\pi}_{n}\right|=\left|J\left(w_{0}\right)\right|\left|s_{n}\right| \text {. }
$$

As a consequence of condition $C\left(\left\{s_{n}\right\}, w_{0}\right)$, there exist two parallelograms $\pi_{n}^{\prime}, \pi_{n}^{\prime \prime}$ each with center at $z_{0}$ and each similar to $\tilde{\pi}_{n}$ with respect to $z_{0}$ (see Figure 1), for which the following statements hold. If $\sigma_{n}$ denotes the closed strip bounded by the perimeters of $\pi_{n}^{\prime}$ and $\pi_{n}^{\prime \prime}$, and if $b_{n}$ denotes the perimeter of $s_{n}$ then

$$
\left\{\begin{array}{l}
T\left(b_{n}\right) \subset \sigma_{n}, \\
\lim \left|\sigma_{n}\right| /\left|s_{n}\right|=0 .
\end{array}\right.
$$

Further, we have for large $n$ (cf. $\$ 2.7$ )

$$
\mu\left(z, T, s_{n}\right)=\mu\left(z, \tilde{T}, s_{n}\right)=\left\{\begin{array}{l}
\operatorname{sgn} J\left(w_{0}\right) \text { if } z \in \pi_{n}^{\prime}, \\
0 \text { if } z \text { not } \in \pi_{n}^{\prime \prime} .
\end{array}\right.
$$

II. Suppose, second, that $J\left(w_{0}\right)=0$. Then $\tilde{T}\left(s_{n}\right)$ reduces to a straight seg-

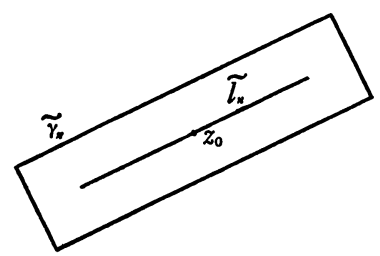

FIG. 2

ment $\tilde{l}_{n}$ with center at $z_{0}$. There exists a closed rectangle $\bar{r}_{n}$ with center at $z_{0}$ (see Figure 2) containing $\tilde{l}_{n}$ in its interior and such that

$$
\begin{gathered}
\left\{\begin{array}{l}
T\left(b_{n}\right) \subset \bar{r}_{n}, \\
\lim \left|\bar{r}_{n}\right| /\left|s_{n}\right|=0,
\end{array}\right. \\
\mu\left(z, T, s_{n}\right)=\mu\left(z, \tilde{T}, s_{n}\right)=0 \text { if } z \text { not } \in \bar{r}_{n} .
\end{gathered}
$$

5.5. Consider now a continuous transformation $T \in K_{3}(\mathcal{D})$ (cf. $\$ 1.37$ ). For almost every point $w_{0} \in \mathcal{D}$ the following statements are true: (i) $D_{1}\left(w_{0}\right)$, $D_{2}\left(w_{0}\right)$ both exist, and $D_{1}\left(w_{0}\right)=D_{2}\left(w_{0}\right)$ (cf. $\left.\S 4.6\right)$; (ii) $J\left(w_{0}\right)$ exists (by assumption); (iii) there exists a sequence $\left\{s_{n}\right\}$ of squares such that the condition $C\left(\left\{s_{n}\right\}, w_{0}\right)$ is satisfied (cf. $\$ 5.3$ ); (iv) $w_{0}$ is not in the subset of $\mathcal{D}-\mathcal{N}$ where $D_{2}>0$ (cf. $\$ \$ 1.18,3.17$, Corollary b in $\$ 3.16$ ). Consider such a point $w_{0}$. I. Assume, first, that $J\left(w_{0}\right) \neq 0$. Then we have conditions as described in I of $\$ 5.4$ and pictured in Figure 1. It follows from (39) and from $\$ 2.21$ that

$$
\mu\left(z, T, s_{n}\right)=\nu\left(z, T, s_{n}^{0}\right) \text { for a.e. } z \text { not } \in \sigma_{n} .
$$


From (40) and (43) it follows (cf. §4.2) that

$$
g_{1}\left(z, T, s_{n}\right)=\left\{\begin{array}{l}
1 \text { a.e. in } \pi_{n}^{\prime}, \\
0 \text { a.e. not in } \pi_{n}^{\prime \prime} .
\end{array}\right.
$$

Hence (cf. §4.3) we have $\left|\pi_{n}^{\prime}\right| \leqq G_{1}\left(s_{n}\right) \leqq\left|\pi_{n}^{\prime \prime}\right|$ and consequently

$$
\left|\tilde{\pi}_{n}\right|-\left|\sigma_{n}\right|<G_{1}\left(s_{n}\right)<\left|\tilde{\pi}_{n}\right|+\left|\sigma_{n}\right| \text {. }
$$

Using (38) in $\$ 5.4$ and conditions (i) and (ii) described in this section, we find from (44) that $D_{1}\left(w_{0}\right)=\left|J\left(w_{0}\right)\right|$, and hence

$$
D_{2}\left(w_{0}\right)=\left|J\left(w_{0}\right)\right| \text {. }
$$

Thus, in particular, $D_{2}\left(w_{0}\right)>0$. In view of condition (iv) of this section, it follows that $w_{0} \in \mathcal{N}$. Consequently we have $j\left(w_{0}\right)=\mu\left(z_{0}, T, s_{n}\right)$ for all $n$ sufficiently large (cf. \$1.19). But then, in view of (40) and (45) it follows that

$$
\mathcal{F}\left(w_{0}\right)=J\left(w_{0}\right) \text {. }
$$

II. Assume, second, that $J\left(w_{0}\right)=0$. Then we have conditions as described in II of $\$ 5.4$ and as pictured in Figure 2. It follows from (41) and from $\$ 2.21$ that

$$
\mu\left(z, T, s_{n}\right)=\nu\left(z, T, s_{n}^{0}\right) \text { for a.e. } z \text { not } \in \bar{r}_{n} .
$$

Thus, from (42) and (47) it follows (cf. $\$ 4.3)$ that $G_{1}\left(s_{n}\right) \leqq\left|\bar{r}_{n}\right|$. Consequently, in view of $(41)$ we have $D_{1}\left(w_{0}\right)=0$. Using conditions (i) and (ii) of this section, we find that

$$
\mathcal{F}\left(w_{0}\right)=0=J\left(w_{0}\right) .
$$

In view of (46) and (48) we have $\mathcal{F}(w)=J(w)$ for every point $w$ which satisfies conditions (i)-(iv) described at the beginning of this section. Since the set of points in $\mathcal{D}$ which fail to satisfy these conditions is of measure zero, the theorem in $\$ 1.38$ is established.

5.6. In order to prove the closure theorem stated in $\$ 1.41$, we need the following

Lemma. Assume that the continuous transformation $T(c f . \S 5.1)$ is such that $\kappa(z, T, \mathcal{D})$ is summable and $J(w)$ exists a.e. in $\mathcal{D}$. Then $D_{2}(w) \geqq|J(w)|$ a.e. in $\mathcal{D}$.

Proof. As a consequence of these assumptions, the following conditions hold for almost every point $w_{0} \in \mathcal{D}$ : (i) $J\left(w_{0}\right), D_{2}\left(w_{0}\right), D_{3}^{*}\left(w_{0}\right)$ exist, and $D_{2}\left(w_{0}\right)=D_{3}^{*}\left(w_{0}\right)$ (cf. $\left.\$ 4.5\right)$; (ii) there exists a sequence $\left\{s_{n}\right\}$ of squares such that the condition $C\left(\left\{s_{n}\right\}, w_{0}\right)$ is satisfied (cf. $\left.\S 5.3\right)$. Let $w_{0}$ be such a point. If $J\left(w_{0}\right)=0$, the lemma is obvious. So assume that $J\left(w_{0}\right) \neq 0$. Then we have conditions as described in I of $\$ 5.4$ and pictured in Figure 1. In view of (40) we have $\kappa\left(z, T, s_{n}^{0}\right) \geqq 1$ for $z$ in $\pi_{n}^{\prime}$ (cf. $\$ 2.7$ ). Consequently (cf. $\$ 4.3$ ), for every $n$, 


$$
G_{3}^{*}\left(s_{n}\right)=\iint \kappa\left(z, T, s_{n}^{0}\right) \geqq\left|\pi_{n}^{\prime}\right|>\left|\tilde{\pi}_{n}\right|-\left|\sigma_{n}\right|
$$

The lemma now follows by condition (i) above, and by (38) and (39) in $\$ 5.4$.

5.7. We prove presently the closure theorem for the class $K_{3}(\mathcal{D})$ stated in $\$ 1.41$. In view of the closure theorem for the class $K_{2}(\mathcal{D})$ stated in $\$ 1.35$ it is sufficient to prove that $T \in K_{3}\left(R^{0}\right)$ for every rectangle $R \subset \mathcal{D}$; for then we can infer from $\$ 1.38$ that $\mathcal{F}(w)=J(w)$ a.e. in $\mathcal{D}$, and consequently the closure theorem for the class $K_{3}(\mathcal{D})$ follows directly from the closure theorem for the class $K_{2}(\mathcal{D})$.

Let $R$ be any rectangle in $\mathcal{D}$. Since $\mathcal{F}\left(w, T_{n}\right)=J\left(w, T_{n}\right)$ a.e. in $\mathcal{D}$ (cf. $\S \S 1.38$, $5.5)$, we have, from the assumptions in the theorem,

$$
\lim \iint_{R}\left|J(w, T)-\mathcal{F}\left(w, T_{n}\right)\right|=0,
$$

and hence

$$
\lim \iint_{R^{0}}\left|\mathcal{F}\left(w, T_{n}\right)\right|=\iint_{R^{0}}|J(w, T)|
$$

Furthermore (cf. \$2.18), we have $\kappa\left(z, T, R^{0}\right) \leqq \lim$ inf $\kappa\left(z, T_{n}, R^{0}\right)$. Now $T_{n} \in K_{3}\left(D_{n}\right)$ and hence $\left({ }^{60}\right) T_{n} \in K_{3}\left(R^{0}\right)$ for all $n$ sufficiently large. Thus it follows (cf. §1.37) that

$$
\kappa\left(z, T, R^{0}\right) \leqq \lim \inf N\left(z, T_{n}, \mathcal{E}\left(T_{n}, \mathcal{D}_{n}\right) \cdot R^{0}\right) \text { for a.e. } z,
$$

and, using (49), we have also

$$
\iint N\left(z, T_{n}, \varepsilon\left(T_{n}, \mathcal{D}_{n}\right) \cdot R^{0}\right)=\iint_{R^{0}}\left|\mathcal{F}\left(w, T_{n}\right)\right| \rightarrow \iint_{R^{0}}|J(w, T)| .
$$

By the lemma of Fatou $\left({ }^{61}\right)$, the relations (50) and (51) yield the summability of $\kappa\left(z, T, R^{0}\right)$. Thus, by $\$ 5.6$, it follows that

$$
|J(w, T)| \leqq D_{2}(w, T) \text { a.e. in } R^{0} .
$$

Since the summability of $\kappa\left(z, T, R^{0}\right)$ implies the summability of $N\left(z, T, \mathcal{\zeta} \cdot R^{0}\right)$, it follows that $T$ is B.V. $\mathcal{E}$ in $\mathcal{D}$ (cf. $\S 3.11$ ). Using $\$ 3.14$, the lemma of Fatou, and the relations $(51),(52)$, we have

$$
\begin{aligned}
\iint_{R^{0}}|J(w, T)| & \leqq \iint_{R^{0}} D_{2}(w, T) \leqq \iint N\left(z, T, \mathcal{E} \cdot R^{0}\right) \leqq \iint \kappa\left(z, T, R^{0}\right) \\
& \leqq \lim \inf \iint N\left(z, T_{n}, \mathcal{E}\left(T_{n}, \mathcal{D}_{n}\right) \cdot R^{0}\right)=\iint_{R^{0}}|\mathcal{F}(w, T)|
\end{aligned}
$$

${ }^{(60)}$ Note that $R \subset \mathcal{D}$ and consequently $R \subset \mathcal{D}_{n}$ for all $n$ sufficiently large.

(61) See $\left.{ }^{48}\right)$. 
Hence the sign of equality holds throughout. In particular,

$$
\begin{aligned}
\iint_{R^{0}} D_{2}(w, T) & =\iint N\left(z, T, \varepsilon \cdot R^{0}\right), \\
\iint N\left(z, T, \varepsilon \cdot R^{0}\right) & =\iint \kappa\left(z, T, R^{0}\right) .
\end{aligned}
$$

By $\$ 3.21,(53)$ implies that $T$ is A.C. $\mathcal{E}$ in $R^{0}$. Since we have $N\left(z, T, \mathcal{E} \cdot R^{0}\right)$ $\leqq \kappa\left(z, T, R^{0}\right)$, the relation (54) implies that $N\left(z, T, \mathcal{E} \cdot R^{0}\right)=\kappa\left(z, T, R^{0}\right)$ a.e. Thus $T \in K_{2}\left(R^{0}\right)$ (cf. $\$ 1.34$ ), but since $J(w, T)$ exists a.e. in $\mathcal{D}$ by assumption, we have $T \in K_{3}\left(R^{0}\right)$.

5.8. Let us now assume that the continuous transformation $T$ (cf. $\S 5.1$ ) satisfies a Lipschitz condition in $\mathcal{D}$ in the restricted sense described in $\$ 1.39$. If $s$ be any square comprised in $\mathcal{D}$, then clearly

$$
\left|T\left(s^{0}\right)\right| \leqq \frac{1}{2} \pi L^{2}|s|
$$

and hence, a fortiori (cf. $\$ 4.3$ ),

$$
G_{2}(s)=\left|T\left(\mathcal{E} \cdot s^{0}\right)\right| \leqq \frac{1}{2} \pi L^{2}|s| .
$$

Thus obviously $T \in K_{1}(\mathcal{D})$ (cf. $\$ 1.32$ ). By (55) however, it follows that $T$ is A.C. $\mathcal{D}$ in $\mathcal{D}$-that is, $T$ is absolutely continuous with respect to $\mathcal{D}$ itself as a base set (cf. $\$ 1.23$ ). Hence (cf. $\$ 3.11), N(z, T, \mathcal{D})$ is summable, and consequently the set of points $z$ where $N(z, T, \mathcal{D})=\infty$ is of measure zero. Consequently, for almost every point $z$ it is true that $T^{-1}(z)$ is a finite set, and therefore every essential maximal model continuum of $z$ under $T$ (cf. \$1.16) consists of a single point. So $N(z, T, \mathcal{E} \cdot \mathcal{D})=\kappa(z, T, \mathcal{D})$ a.e. (cf. $\$ 1.18$ ), and hence $T \in K_{2}(\mathcal{D})$ (cf. $\left.\$ 1.34\right)$. But, as a consequence of the restricted Lipschitz condition, the partial derivatives $x_{u}, x_{v}, y_{u}, y_{v}$ exist a.e. in $\mathcal{D}$. So $T \in K_{3}(\mathcal{D})$, as asserted in $\$ 1.39$.

5.9. Finally, suppose that the continuous transformation $T$ (cf. $\S 5.1$ ) is such that $x_{u}, x_{v}, y_{u}, y_{v}$ exist and are continuous everywhere in $\mathcal{D}$, and that the ordinary Jacobian $J(u, v)$ is summable in $\mathcal{D}$. Take a sequence of Jordan regions $\Re_{n}$ which fill up $\mathcal{D}$ from the interior (cf. $\$ 1.13$ ). Clearly $T$ satisfies a restricted Lipschitz condition in $\Re_{n}^{0}$ for every $n$ and hence $T \in K_{3}\left(\Re_{n}^{0}\right)$ by $\S 5.8$. The closure theorem for $K_{3}$ (cf. $\$ 1.41$ ), applied with $T_{n}=T$ and $\mathcal{D}_{n}=\Re_{n}^{0}$, yields immediately the fact that $T \in K_{3}(\mathcal{D})$.

S. BANACH

\section{BIBLIOGRAPHY}

1. Sur une classe de fonctions d'ensemble, Fundamenta Mathematicae, vol. 6 (1924), pp. 170-188.

2. Sur les lignes rectifiables et les surfaces dont l'aire est finite, Fundamenta Mathematicae, vol. 7 (1925), pp. 225-236. 
H. E. Bray

1. Proof of a formula for an area, Bulletin of the American Mathematical Society, vol. 29 (1923), pp. 264-270.

R. Caccioppoli

1. Sulle funzioni additive d'insieme, Rendiconti della R. Accademia di Scienze Fisiche e Matematiche di Napoli, (3), vol. 34 (1927), pp. 150-153.

2. Trasformazioni piane, superficie quadrabili, integrali di superficie, Rendiconti del Circolo Matematico di Palermo, vol. 54 (1930), pp. 217-262.

B. VON KERÉKJÁRTó

1. Vorlesungen über Topologie, vol. 1, Berlin, 1923.

C. KuRATOWSKI

1. Topologie I, Warsaw and Lwow, 1933.

E. J. McShane

1. Integrals over surfaces in parametric form, Annals of Mathematics, (2), vol. 34 (1933), pp. 815-838.

C. B. MORREY

1. A class of representations of manifolds, part I, American Journal of Mathematics, vol. 55 (1933), pp. 683-707.

H. RADEMACHER

1. Über partielle und totale Differenzierbarkeit von Funktionen mehrerer Variabeln, parts I and II, Mathematische Annalen, vol. 79 (1919), pp. 340-359, and vol. 81 (1920), pp. $52-63$.

T. RADó

1. Über das Flächenmass rektifizierbarer Flächen, Mathematische Annalen, vol. 100 (1928), pp. 445-479.

2. On continuous transformations in the plane, Fundamenta Mathematicae, vol. 27 (1936), pp. 201-211.

3. A remark on the area of surfaces, American Journal of Mathematics, vol. 58 (1936), pp. 598-606.

4. On the derivative of the Lebesgue area of continuous surfaces, Fundamenta Mathematicae, vol. 30 (1938), pp. 34-39.

5. On absolutely continuous transformations in the plane, Duke Mathematical Journal, vol. 4 (1938), pp. 189-221.

S. SAKS

1. Theory of the Integral, New York, Stechert, 1937.

J. Schauder

1. Über stetige Abbildungen, Fundamenta Mathematicae, vol. 12 (1928), pp. 47-74. K. SzILÁRD

1. Untersuchungen über die Grundlagen der Funktionentheorie, Mathematische Zeitschrift, vol. 26 (1927), pp. 653-671.

C. DE la Vallée Poussi,n

1. Sur l'intégrale de Lebesgue, these Transactions, vol. 16 (1915), pp. 435-501.

W. H. Young

1. On a formula for an area, Proceedings of the London Mathematical Society, vol. 18 (1920), pp. 339-374.

2. On a new set of conditions for a formula for an area, Proceedings of the London Mathematical Society, vol. 21 (1923), pp. 75-94.

3. Integration over the area of a curve and the transformation of the variables in a multiple integral, Proceedings of the London Mathematical Society, vol. 21 (1923), pp. 161-190.

The Ohio State University, Columbus, OHio. 